\title{
The Einstein Equations of Evolution-A Geometric Approach
}

\author{
Arthur E. Fischer* and Jerrold E. Marsden ${ }^{\dagger}$ \\ Department of Mathematics, University of California, Berkeley, California 94720 \\ (Received 12 July 1971)
}

In this paper the exterior Einstein equations are explored from a differential geometric point of view. Using methods of global analysis and infinite-dimensional geometry, we answer sharply the question: "In what sense are the Einstein equations, written as equations of evolution, a Lagrangian dynamical system?" By using our global methods, several aspects of the lapse function and shift vector field are clarified. The geometrical significance of the shift becomes apparent when the Einstein evolution equations are written using Lie derivatives. The evolution equations are then interpreted as evolution equations as seen by an observer in space coordinates. Using the notion of body-space transitions, we then find the relationship between solutions with different shifts by finding the flow of a time-dependent vector field. The use of body and space coordinates is shown to be somewhat analogous to the use of such coordinates in Euler's equations for a rigid body and the use of Eulerian and Lagrangian coordinates in hydrodynamics. We also explore the geometry of the lapse function, and show how one can pass from one lapse function to another by integrating ordinary differential equations. This involves integrating what we call the "intrinsic shift vector field." The essence of our method is to extend the usual configuration space $\mathbb{T}=\operatorname{Riem}(M)$ of Riemannian metrics to $T \times \mathbb{D} \times \mathbb{T}$, where $T=C^{\infty}(M, \mathbf{R})$ is the group of relativistic time translations and $D=\operatorname{Diff}(M)$ is the group of spatial coordinate transformations of $M$. The lapse and shift then enter the dynamical picture naturally as the velocities canonically conjugate to the configuration fields $\left(\xi_{t}, \eta_{t}\right) \in T \times D$. On this extended configuration space, a degenerate Lagrangian system is constructed which allows precisely for the arbitrary specification of the lapse and shift functions. We reinterpret a metric given by DeWitt for $\mathfrak{N}$ as a degenerate metric on $\mathfrak{D} \times \mathfrak{M}$. On $\mathfrak{D} \times \mathfrak{M}$, however, the metric is quadratic in the velocity variables. The groups $T$ and $D$ also serve as symmetry groups for our dynamical system. We establish that the associated conserved quantities are just the usual "constraint equations." A precise theorem is given for a remark of Misner that in an empty space-time we must have $\mathcal{H}=0$. We study the relationship between the evolution equations for the time-dependent metric $g_{t}$ and the Ricci flat condition of the reconstructed Lorentz metric $g L$. Finally, we make some remarks about a possible "superphase space" for general relativity and how our treatment on $\mathcal{T} \times \mathbb{D} \times \mathfrak{T}$ is related to ordinary superspace and superphase space.

\section{INTRODUCTION: THE EINSTEIN EQUATIONS OF EVOLUTION}

Our aim in this paper is to study the Einstein equations of evolution as a dynamical system, to explore the geometrical meaning of the lapse function $N$ and shift vector field $X$ introduced by Wheeler, ${ }^{1}$ to introduce the gauge groups $T$ and $D$ appropriate for the dynamical formulation of general relativity, and to study the interrelationships of the evolution equations with $N$ and $X$, with the four-dimensional empty space condition $R_{\alpha \beta}=0$, and with the gauge groups $\widetilde{T}$ and $D$. The gauge groups $T$ and $D$ and the meaning of $N$ and $X$ are explained below.

Basic work on the problem of regarding the Einstein equations of evolution as a dynamical system has been done by Arnowitt, Deser, and Misner, ${ }^{2}$ by DeWitt, ${ }^{3}$ and by Wheeler. ${ }^{1}$ We shall reformulate this work using the general theory of Lagrangian systems and exploiting differential geometric ideas. We shall also be extending this work and viewing it in a way which differs from the original approaches in several essential ways. Perhaps the most fundamental of these ways may be explained as follows: One usually fixes a three-dimensional manifold $M$ (taken to be a spacelike hypersurface in the final space-time) and uses as configuration space the space $\mathrm{m}$ of all Riemannian metrics on $M$. To incorporate the lapse function $N$ and shift vector field $X$ in what we believe is a natural way, we have found it necessary to enlarge $\mathfrak{N}$ to $\mathcal{T} \times \mathcal{D} \times \mathfrak{T}$, where

$\tau=C \infty(M ; R)=$ all smooth real-valued functions, $\xi: M \rightarrow \mathbf{R}$, which one can think of as the "relativistic time translation group"

and

$D=\operatorname{Diff}(M)=$ all diffeomorphisms $\eta: M \rightarrow M$, which one can think of as the "active" coordinate transformations or the "rotation group" of $M$.

The sense in which $\mathcal{T}$ is the relativistic time translation group and in which $D$ is the rotation group of $M$ is described briefly below and in detail in Secs. 7 and
5 , respectively. The groups $T$ and $D$ are closely related to the lapse and shift as we shall explain shortly.

The lapse $N$, a real valued function, represents the clock rates for an observer relative to a reference system of clocks. The clock rates $N$ depend on the space-time point for the observer. The fact that we change our clock rates, that is, allow an $N$ not identically one, changes the equations of motion for the 3metric $g_{i j}$ which describes the geometry of the space $M$ (the equations are written out below). Similarly a shift $X$ is a vector field on the 3-manifold $M$ which represents two observers in relative motion with velocity described by $X$. Again, a choice $X \neq 0$ will change the equations of motion.

The introduction of $\tau \times D$ is essentially the introduction of the configuration variables $(\xi, \eta)$, whose canonically conjugate velocities are the lapse $N$ and shift $X$ (when the tangent space to $\tau \times D$ is "pulled back" to the identity; see Secs. 4 and 7 for a description of this process). On $T \times D \times M$ we construct an infinite-dimensional degenerate Lagrangian system $L: T(T \times D \times I T) \rightarrow \mathbf{R}$. The degeneracy is, roughly speaking, in the direction of $\mathcal{T} \times D$. The degeneracy allows precisely for the arbitrary specification of the lapse function and shift vector field.

In our approach, we also consider the geometrical significance of the lapse and shift in the equations of evolution. In the treatment of Arnowitt, Deser, and Misner, ${ }^{2}$ the lapse and shift are incorporated into the Lagrangian on $\mathfrak{T}$ as Lagrange multipliers. The constraint equations (see below) are then obtained by varying the lapse and shift. In our formulation this situation is rather different. We consider the lapse and shift as velocities canonically conjugate to some configuration field variables rather than as Lagrange multipliers. The degeneracy of our Lagrangian on $\tau \times \mathbb{D} \times \mathbb{T}$ allows an arbitrary lapse and shift to be consistent with the equations of motion.

We consider the two basic constraints of the field equations, namely the divergence constraint

$$
\delta((\operatorname{Tr} k) g-k) \mu_{g}=0
$$


and the Hamiltonian constraint

$$
\mathcal{F}(g, k) \mu_{g} \equiv \frac{1}{2}\left((\operatorname{Tr} k)^{2}-(k \cdot k)\right) \mu_{g}+2 R(g) \mu_{g}=0
$$

(see below for definitions) as conservation laws rather than as "constraints." We shall show (see Sec. 6 ) that conservation in time of (1.1) is a result of the invariance of the evolution equations under the coordinate symmetry group D. (1.2) is first established in Sec. 3 under the hypothesis that $\delta \pi=0$ by a straightforward computation. What is interesting, however, is that we shall show that the pointwise conservation of $\mathcal{H} \mu_{g}$ is a necessary consequence of the "full relativistic invariance" of the theory (in a sense made precise in Sec. 7). Our theorem is a rigorous version of remarks of Misner 4,2 that a "topologically invariant" theory must have an identically zero Hamiltonian.

Note: Our Lagrangian on $\tau^{*} \times \mathscr{D} \times \mathfrak{T}$ is homogeneous and degenerate and our assertion about $\mathcal{H} \mu_{g}$ is distinct from and not to be confused with the elementary remark (see Ref. 5) that the energy of a homogeneous Lagrangian is always zero, as $\mathcal{H}$ is the energy of the Lagrangian before it is made homogeneous. We also remark that the infinite dimensionality of the invariance groups leads to pointwise integrals of the motion rather than integrated conserved quantities which one normally obtains.

We now formally write out the Einstein system for a given lapse $N$ and shift $X$. It is important for the later geometrical development that certain combinations of the terms be recognized as Lie derivatives and Hessians.

Note: In the following, $t$ occurring as a subscript indicates the variable $t$; it is never used to denote differentiation. Often the time-dependence of a field will be implicit.

The Einstein System $(E)$ : Let $X_{t}$ be a time-dependent vector field on a fixed compact orientable threedimensional manifold $M$, and let $N_{t}$ be a time-dependent positive real valued (scalar) field on $M$, that is, $N_{t}(m)>0$ for all $m \in M$ and $t \in \mathbf{R}$. The Einstein system is the system of evolution equations

$$
\left\{\begin{array}{l}
\frac{\partial g_{t}}{\partial t}=N_{t} k_{t}-L_{X_{t}} g_{t}, \\
\frac{\partial k_{t}}{\partial t}=N_{t} S_{g_{t}}\left(k_{t}\right)-2 N_{t} \operatorname{Ric}\left(g_{t}\right)+2 \operatorname{Hess}\left(N_{t}\right)-L_{X_{t}} k_{t}
\end{array}\right.
$$

with the supplementary conditions

$$
\left\{\begin{aligned}
\delta\left(\left(\operatorname{Tr} k_{t}\right) g_{t}-k_{t}\right) & =0, \\
\nVdash\left(g_{t}, k_{t}\right) & =\frac{1}{2}\left(\left(\operatorname{Tr} k_{t}\right)^{2}-k_{t} \cdot k_{t}\right)+2 R\left(g_{t}\right)=0 .
\end{aligned}\right.
$$

\section{Our notation is the following:}

$$
\begin{aligned}
& g_{t} \quad \text { is a time-dependent metric on } M \text {, } \\
& L_{X_{t}} g_{t} \quad=\text { Lie derivative of } g_{t} \text { with respect to the } \\
& \begin{aligned}
L_{X_{t}} k_{t}= & \text { Lie derivative of } k_{t}=X^{\imath} k_{i j \mid l}+k_{i l} X_{\mid j}^{l} \\
& +k_{j l} X^{l},
\end{aligned}
\end{aligned}
$$

$$
\begin{aligned}
\operatorname{Ric}\left(g_{t}\right)= & \text { (Ricci curvature tensor formed from } \left.g_{t}\right) \\
& =R_{i j}=\Gamma_{i j, k}^{k}-\Gamma_{k i, j}^{k}+\Gamma_{i j}^{k} \Gamma_{k l}^{l}-\Gamma_{i k}^{l} \Gamma_{l j}^{k}, \\
R\left(g_{t}\right)= & \text { scalar curvature }=R_{k}^{k}, \\
\text { Hess }(N)= & \text { Hessian of } N=\text { double covariant deriva- } \\
& \text { tive }=N_{|i| j}, \\
\delta k & \text { divergence of } k=(\delta k)_{i}=-k_{i}{ }^{j}{ }_{j}, \\
\operatorname{Tr} k \quad & \text { trace } k=g^{i j} k_{i j}=k_{i}^{i}, \\
k \cdot k \quad & \text { dot product for symmetric tensors }=k_{i j} k^{i j}, \\
k \times k= & \text { cross product for symmetric tensors } \\
= & k_{i l} k_{j}^{l}, \\
S_{g}(k)= & k \times k-\frac{1}{2}(\operatorname{Tr} k) k=k_{i l} k_{j}^{l}-\frac{1}{2}\left(g^{m n} k_{m n}\right) k_{i j} .
\end{aligned}
$$

Note: We have assumed $M$ compact only to simplify the discussions. It is surely not essential.

The Lorentz geometry on $(-\epsilon, \epsilon) \times M$ corresponding to a solution $g_{t}=3 g_{t}$ of the Einstein system is given by

${ }^{4} g_{\alpha \beta} d x^{\alpha} d x^{\beta}=\left(X^{i} X_{i}-N^{2}\right) d t^{2}-2 X_{i} d x^{i} d t+g_{i j} d x^{i} d x^{j}$,

where $x^{\alpha}=\left(t, x^{i}\right)$ and $X^{i} X_{i}={ }^{3} g(X, X)$. For this to be Lorentz, we require ${ }^{3} g(X, X)<N^{2}$ initially (and hence for a short $t$ interval).

Our conventions in this paper will be that, for expressions written in coordinates, Latin indices will run from 1 to 3 and Greek indices from 0 to 3 . Our Lorentz metrics will have signature $(-,+,+,+)$.

In the Einstein system, all the geometric quantities (such as Ric, Hess, $\delta$, and $\mathrm{Tr}$ ) are computed with respect to the time-dependent metric $g_{t}$. These equations appear in coordinates in Arnowitt, Deser, and Misner (see Ref.2,p. 236 or Ref.6, p. 1325) in terms of the tensor density $\pi=((\operatorname{Tr} k) g-k) \sqrt{\operatorname{det} g} d x^{1} \wedge d x^{2} \wedge d x^{3}$ (using the momentum rather than the velocity variable). $k$, our energy density (the second of the supplementary conditions), and the second evolution equation are minus twice the corresponding quantities in Ref. 6 , and our shift is minus theirs. Our change of numerics makes the system more manifestly a second-order system with $\partial g_{t} / \partial t=k_{t}$ when $N=1$, $X=0$ (see Sec. 3); changes the energy to the form $K+V$, where $V$ is now the integrated scalar curvature (and not its negative; see DeWitt ${ }^{3}$ and Sec. 3 ); and introduces a factor of $\frac{1}{2}$ into the kinetic energy part $\pi$ of the energy density. The reason for changing the sign of the shift is explained at the end of Sec. 4.

Unfortunately, the Einstein system, when written in coordinates, obscures the central role played by the presence of the Lie derivative in the evolution equations. In fact, the apparent complexity of the equations as they appear in Refs. 2 or 6 dissolves when it is recognized that the last five terms in Ref. 6 or the last three terms in Ref. 2 are just the Lie derivative of either $k$ or $\pi$ (see also remarks in Sec. 6). Thus, when written intrinsically, the Einstein system is geometrically simplified.

The Lie derivative terms have a natural geometric interpretation related to changing from "space" to "body" coordinates in a manner similar to that of the rigid body and hydrodynamics (cf. Ref. 7). This may 
be more specifically described as follows. The shift $X_{t}$ is a time-dependent vector field on $M$, and as such it has an integral, or flow $\eta_{t} \in \mathcal{D}$ with $\eta_{0}=$ (identity diffeomorphism of $M$ ) $=i d_{M}$. The solutions of the Einstein system with the shift $X$ and those with the shift zero are related by the active coordinate transformation $\eta_{t}$ in the usual way one transforms metrics. Moreover, we consider the manifold $M$ to be the "body" and the flow $\eta_{t}$ of the shift vector field as being a motion or "rotation" of $M$. Then if we assume that the time-dependent metric field $g_{t}$ is "dragged along" by the rotation of $M$, the Einstein system can be interpreted as the equations of evolution as seen by an observer fixed in space, taken to be "off" the rotating manifold. This interpretation is worked out in Secs. 4 and 5.

The lapse $N_{t}$ enters the evolution equations in a slightly more subtle way, as it involves a system of clocks on $M$ whose rates may be different at different points of $M$. The complication due to the possible space-dependence of $N_{t}$ is reflected in the Hessian term in the Einstein system (E).

As for the shift vector field we show how to solve the Einstein equations for a general lapse $N$, given the solution for $N=1$, again by integrating a system of ordinary differential equations. Conversely, given a solution $g$ for a given lapse $N$, we shall show how to construct a vector field on $M$, called the intrinsic shift of the lapse $N$, whose flow, together with a proper time function, brings us into a Gaussian normal coordinate system in which $N=1$. The intrinsic shift vector field may be interpreted as a "sliding effect" due to the fact that $N_{t}$ is not constant in the space variable.

Finally, we remark that on $D \times \mathfrak{M}$, our Lagrangian is quadratic in the velocities $(X, \partial g / \partial t)$ and is therefore of the classical form-kinetic energy minus potential energy, with the kinetic energy being derived from a degenerate metric on $D \times M T$. In fact the evolution equation $\partial k / \partial t=k \times k-\frac{1}{2}(\operatorname{Tr} k) k-L_{X} k$, along a solution for which the kinetic energy $=0$, is just the geodesic equation on $D \times M$ with respect to the aforementioned metric. When written just on $\mathbb{N}$, the full Lagrangian does not have this classical form.

We now summarize the topics treated in this paper:

1. A treatment of infinite-dimensional degenerate Lagrangian systems (Sec. 2). A basic conservation law is given, similar to the nondegenerate case, which generalizes the classical conservation laws, 8,9,11

2. The introduction of the gauge groups $\mathcal{T}$ and $\mathfrak{D}$ and of the space $\mathcal{T} \times \mathbb{D} \times \mathfrak{K}$ as the configuration space for the Einstein system. The gauge groups $T$ and $D$ are the analog for the dynamical formulation of the coordinate gauge group of the four-dimensional geometry.

3. A geometrical interpretation of the lapse and shift functions as the velocities canonically conjugate to the new configuration variables $(\xi, \eta) \in \mathcal{T} \times \mathbb{D}$.

4. A treatment of the Einstein system as an infinitedimensional degenerate Lagrangian system on a suitable subset of $\widetilde{T} \times \mathfrak{D} \times \mathfrak{T}$ (Secs. 2 and 7). On $\mathfrak{D} \times \mathfrak{T}$ we construct a Lagrangian $L=K-V$, where $K$ is quadratic in the velocities $(X, h)$, and on $T \times D \times \mathscr{T}$ a Lagrangian homogeneous in the velocities $(N, X, h)$.
The quadratic nature of the Lagrangian on $\mathbb{D} \times \mathfrak{M}$ results in evolution equations quadratic in the velocities $(X, h)$ which is analagous to the quadratic nature of the evolution equations for geodesics on a manifold and for hydrodynamics and the rigid body.

5. A derivation of the supplementary conditions for the Einstein system as conserved quantities (Secs. 3 and 10), using conservation laws for degenerate Lagrangian systems and infinite-dimensional symmetry groups $(T$ and $D)$, together with a precise explanation of why the energy density for the empty space-time equations must be identically zero; cf. Misner. ${ }^{4}$

6. The recognition of the central role played by the Lie derivative terms in the evolution equations. The use of Lie derivatives shows that these terms in the evolution equations are geometrically simple and makes their geometric meaning transparent.

7. An interpretation of the shift vector field $X_{t}$ as generating a "rotation" of $M$ and of the evolution equations as being the equations as seen by an observer in space coordinates. Using the notion of bodyspace transitions, we then show that if we can solve the equations for $X=0$, then they can be solved for any $X$ by integrating a system of ordinary differential equations (Corollary 4.1). Similarly, we give a geometrical derivation of how if one can solve the Einstein equations for $N=1$, then they can be solved for an arbitrary $N$ by integrating geodesic equations, again ordinary differential equations (Theorem 10.3).

8. A geometrical derivation of the Hessian term Hess $(N)$ in the Einstein system using generalized Gauss-Codazzi equations in coordinates which are not necessarily normal Gaussian (Sec. 8).

9. The interpretation of the lapse $N_{t}$ as related to the tangent of the curve $\tau_{t} \in T$ where $\tau_{t}$, the proper time function, can be interpreted as a change of time parameter for each point $m \in M$ from the canonical parameter of evolution to an arbitrary parameter of evolution (Sec. 10).

10. The introduction of a new object, the intrinsic shift $Y$ of $N$, whose integration gives the rest of the Gaussian coordinate system (Theorems 10.1 and 10.2). The intrinsic shift is interpreted as the "tilting effect" of the coordinate system due to the spatial dependence of $N$.

\section{DEGENERATE LAGRANGIAN SYSTEMS}

In this section we study the notion of a degenerate Lagrangian system in the spirit of Ref. 8. Degenerate Lagrangian systems have been used in some previous analyses of general relativity and are fairly common in classical mechanics (cf. Ref.9). Here we shall treat such systems from the coordinate independent or global point of view.

We are going to be working with spaces of maps, for example, the space of all Riemannian metrics Ti. For simplicity we assume that all such objects are $C^{\infty}$. Properly one should work with Sobolev spaces, but the modifications needed are fairly routine and do not involve any new physical ideas (see Refs. 10 and 11).

For our purposes we find it convenient to use the general ideas about Hamiltonian and Lagrangian systems as developed in Ref. 8 rather than variational 
principles. The chief difference with Ref. 8 is that we must use infinite-dimensional configuration spaces. We, therefore, shall assume that the reader has some aquaintance with calculus in infinite dimensional spaces and manifolds, as expounded for example in Ref.12. As mentioned above, the spaces are usually spaces of maps; in the physics literature the derivative of a function defined on such a space is often called its "functional derivative."

Let $B$ be a manifold, possibly infinite-dimensional (modeled on a Fréchet or Banach space). Let $T B$ denote its tangent bundle and let $\tau: T B \rightarrow B$ be the natural projection map. For $b \in B$, let $T_{b} B$ be the tangent space at $b$, that is, the fiber over $b ; T_{b} B=$ $\tau^{-1}(b)$.

Let $L: T B \rightarrow \mathbf{R}$ be a mapping (called the Lagrangian or action integral) and let $L_{b}$ be the restriction of $L$ to $T_{b} B$. The derivative of $L_{b}, D L_{b}(v)$, at a point $v \in T_{b} B$ defines a map $F L: T B \rightarrow T^{*} B\left(T^{*} B\right.$ is the contangent bundle), called the fiber derivative or Legendre transformation. As in Ref. 8, using $F L$, one obtains a closed 2 -form $\omega_{L}$ (i.e., $d \omega_{L}=0$ ) on $T B$ by pulling back the canonical symplectic structure on $T^{*} B$. If $B$ is modeled on a linear space $\mathbf{E}$, so locally $T B$ looks like $U \times \mathbf{E}$ where $U \subset \mathbf{E}$ is open, then $\omega_{L}(u, e)$, for $(u, e) \in U \times \mathbf{E}$, is a skew-symmetric bilinear form on $\mathbf{E} \times \mathbf{E}$ given by

$$
\begin{aligned}
2 \omega_{L}(u, e) \cdot\left(\left(e_{1}, e_{2}\right),\left(e_{3}, e_{4}\right)\right) & \\
= & D_{1}\left(D_{2} L(u, e) \cdot e_{1}\right) \cdot e_{3}-D_{1}\left(D_{2} L(u, e) \cdot e_{3}\right) \cdot e_{1} \\
& +D_{2} D_{2} L(u, e) \cdot e_{4} \cdot e_{1}-D_{2} D_{2} L(u, e) \cdot e_{2} \cdot e_{3},
\end{aligned}
$$

where $D_{1}, D_{2}$ denote the partial derivatives of $L$. We say $\omega_{L}$ is (weakly) nondegenerate if $\omega_{L}(u, e)$. $\left(\left(e_{1}, e_{2}\right),\left(e_{3}, e_{4}\right)\right)=0$ for all $e_{3}, e_{4} \in \mathbf{E}$ implies that $\left(e_{1}, e_{2}\right)=0$. However we will want to allow for degenerate $\omega_{L}$, so we do not make this assumption. We say that $L$ is degenerate if $\omega_{L}$ is degenerate (as a 2 -form). It is easy to see that $\omega_{L}$ is nondegenerate iff $D_{2} D_{2} L(u, e)$ is nondegenerate.

The action of $L$ is defined by $A: T B \rightarrow \mathbf{R}, A(v)=$ $F L(v) \cdot v$, and the energy of $L$ is $E=A-L$. In charts,

$$
E(u, e)=D_{2} L(u, e) \cdot e-L(u, e)
$$

and in finite dimensions it is the usual expression

$$
E(q, \dot{q})=\frac{\partial L}{\partial \dot{q}^{i}} \dot{q}^{i}-L(q, \dot{q}) .
$$

Now given $L$, we say that a vector field $Z$ on $T B$ is a Lagrangian vector field or a Lagrangian system for $L$ if the Lagrangian condition holds:

$$
2 \omega_{L}(v)(Z(v), w)=d E(v) \cdot w
$$

for all $v \in T_{b} B, w \in T_{v}(T B)$. Here $d E$ denotes the differential of $E$.

If $\omega_{L}$ were a (weak) symplectic form, i.e., nondegenerate, there would be at most one such $Z$. The fact that $\omega_{L}$ is degenerate however means that $Z$ is not uniquely determined by $L$ so that there is some arbitrariness in what we may choose for $Z$. For the Einstein system, this degeneracy will correspond to an arbitrary choice of lapse and shift.
It should also be stressed that, in general, $L$ need not have a corresponding $Z$. If there is one, we say that we can find consistent equations of motion for $L$. As above, there can be several equations of motion consistent with $L$.

The dynamics is obtained by finding the integral curves of $Z$; that is, the curves $v(t)$ such that $v(t) \in T B$ satisfies $(d v / d t)(t)=Z(v(t))$. From the Lagrangian condition, it is trivial to check that energy is conserved even though $L$ may be degenerate.

Proposition 2. 1: Let $Z$ be a Lagrangian vector field for $L$ and let $v(t) \in T B$ be an integral curve of $Z$. Then $E(v(t))$ is constant in $t$.

Proof: By the chain rule,

$$
\begin{aligned}
\frac{d}{d t} E(v(t)) & =d E(v(t)) \cdot v^{\prime}(t)-d E(v(t)) \cdot Z(v(t)) \\
& =2 \omega_{L}(v(t))(Z(v(t)), Z(v(t))) \\
& =0 \text { by the skew symmetry of } \omega_{L}
\end{aligned}
$$

For a general degenerate Lagrangian system, Lagrange's equations also hold, if we assume that $Z$ is second order. That $Z$ is second order means that, in a chart $U \times \mathbf{E}, Z$ has the form $Z(u, e)=\left(e, Z_{2}(u, e)\right)$. (See Refs. 8 and 12 for the intrinsic definition of second order.)

Proposition 2.2: Let $Z$ be a Lagrangian system for $L$ and suppose $Z$ is a second order equation. Then in the chart $U \times \mathbf{E}$, an integral curve $(u(t), v(t)) \in$ $U \times \mathbf{E}$ of $Z$ satisfies Lagrange's equations:

$$
\left\{\begin{array}{l}
\frac{d u}{d t}(t)=v(t), \\
\left.\frac{d}{d t}\left(D_{2} L(u(t)), v(t)\right) \cdot \omega\right)=D_{1} L(u(t), v(t)) \cdot \omega
\end{array}\right.
$$

for all $\omega \in \mathbf{E}$. In case $L$ is nondegenerate,

$$
\frac{d v}{d \bar{t}}=\left\{D_{2} D_{2} L(u, v)\right\}^{-1}\left\{D_{1} L(u, v)-D_{1} D_{2} L(u, v) \cdot v\right\}
$$

Proof: We work in a chart $U \times \mathbf{E}$ so that $Z(u, e)=$ $\left(e, Z_{2}(u, e)\right)$. From the definition of $E$, we have $d E(u, e) \cdot\left(e_{1}, e_{2}\right)=D_{1}\left(D_{2} L(u, e) \cdot e\right) \cdot e_{1}+$ $D_{2} D_{2} L(u, e) \cdot e \cdot e_{2}-D_{1} L(u, e) \cdot e_{1}$. Using the formula for $\omega_{L}$, the condition on $Z_{2}$ may be written, after a short computation, as

$$
\begin{aligned}
& D_{1} L(u, e) \cdot e_{1}=D_{1}\left(D_{2} L(u, e) \cdot e_{1}\right) \cdot e \\
& \quad+D_{2}\left(D_{2} L(u, e) \cdot Z_{2}(u, e)\right) \cdot e_{1}
\end{aligned}
$$$$
\text { for all } e_{1} \in \mathbf{E} \text {. }
$$

If $(u(t), v(t))$ is an integral curve of $Z$, we obtain (where the dot means $d / d t$ )

$$
\begin{aligned}
D_{1} L(u, v) \cdot e_{1} & =D_{1} D_{2} L(u, \dot{u}) \cdot e_{1} \cdot \dot{u}+D_{2} D_{2} L(u, \dot{u}) \cdot \ddot{u} \cdot e_{1} \\
& =\frac{d}{d t} D_{2} L(u, \dot{u}) \cdot e_{1}
\end{aligned}
$$

by the chain rule.

We wish to emphasise a special case of Proposition 2.2 for later use. Suppose that $\langle$,$\rangle is a symmetric$ bilinear form defined on each tangent space of $B$; we shall refer to $\langle$,$\rangle as a metric. Define L: T B \rightarrow \mathbf{R}$ to 
be the kinetic energy $L(v)=\frac{1}{2}\langle v, v\rangle$. Suppose that the metric is nondegenerate, which implies that the Lagrangian $L$ is also nondegenerate. Then $Z$ is called the geodesic spray and one can easily check from Proposition 2. 2 that in the finite-dimensional case,

$$
L(v)=\frac{1}{2} g_{i j} v^{i} v j \quad \text { and } \quad Z_{2}(u, e)=-\Gamma_{i j}^{k}(u) e^{i} e^{j}
$$

where $\Gamma_{i j}^{k}$ are the Christoffel symbols of the metric $g_{i j}$.

Thus the integral curves of the geodesic spray are given by $v(t)=d x(t) / d t$, where $x(t)$ is a geodesic on $B$. Now let $V: B \rightarrow \mathbf{R}$ be given and consider the Lagrangian $L\left(v_{b}\right)=\frac{1}{2}\left\langle v_{b}, v_{b}\right\rangle-V(b)$. Then from Proposition 2.2 we see that the Lagrangian vector field for $L$ is given by $Z(u, e)=\left(e, S_{2}(u, e)-\operatorname{grad} V(u)\right)$, where $S$ is the spray of the metric and where $\operatorname{grad} V$ is the gradient of $V$, a vector field on $B$ defined by

$$
\left\langle\operatorname{grad} V(b), v_{b}\right\rangle=d V(b) \cdot v_{b},
$$

where $d V(b)$ is the differential of $V$ evaluated at $b$. Again, in the finite-dimensional case, $\operatorname{grad} V=$ $g^{i j} \partial V / \partial x j$.

We remark that if $\omega_{L}$ were nondegenerate, $Z$ would automatically be a second order equation (cf. Ref. 8). But for a general $\omega_{L}$, a Lagrangian vector field $Z$ need not be second order; if it is not, Lagrange's equations may fail-and do in some important examples, such as when a quantum mechanical system is regarded as a Lagrangian system.

Often $L$ is obtained in the form

$$
L(u, \dot{u})=\int_{M} \mathfrak{L}\left(u, \frac{\partial u}{\partial x^{k}}, \dot{u}\right) d x .
$$

Here $M$ is some fixed manifold, say $\mathbf{R}^{n}, u$ is a scalar or possibly tensor field on $M$ and $\mathcal{L}$ is a given scalar function with the indicated arguments. The space of the $u^{\prime} s$ forms the manifold $B$ and the Lagrange's equations can be converted to the usual Lagrange density form.

We next give the basic conservation law for Lagrangian mechanics. A key point is that the validity of the result is not affected by the fact that $L$ may be degenerate.

Proposition 2.3: Let $Z$ be a Lagrangian vector field for $L: T B \rightarrow \mathbf{R}$, and suppose $Z$ is a second-order equation.

Let $\Phi_{t}$ be a one-parameter group of diffeomorphisms of $B$ generated by the vector field $Y: B \rightarrow T B$. Suppose that for each real number $t, L \circ T \Phi_{t}=L$. Then the function $P(Y): T B \rightarrow \mathbf{R}, P(Y)(v)=F L(v) \cdot Y$ is a constant along integral curves of $Z$.

Proof: Let $v(t)$ be an integral curve for $Z$. Then we shall show that $(d / d t) P(Y)(v(t))=0$. Indeed, in a coordinate chart, if $(u(t), v(t))$ is the integral curve, we get from the chain rule

$$
\begin{aligned}
\frac{d}{d t}\{F L(v(t)) \cdot Y\}=\frac{d}{d t}\left\{D_{2} L(u(t), v(t)) \cdot Y(u(t))\right\} \\
=D_{1} D_{2} L(u(t), v(t)) \cdot Y(u(t)) \cdot u^{\prime}(t) \\
\quad+D_{2} D_{2} L(u(t), v(t)) \cdot Y(u(t)) \cdot v^{\prime}(t) \\
\quad+D_{2} L(u(t), v(t)) \cdot D Y(u(t)) \cdot u^{\prime}(t)
\end{aligned}
$$

Now the condition that $Z$ be the Lagrangian vector field of $L$ means exactly that the first two terms equal $D_{1} L(u(t), v(t)) \cdot Y(u(t))$ (see the proof of Proposition 2.2 above). However if we differentiate $L \circ T \Phi_{t}$ with respect to $t$ we obtain for any point $(u, v)$,

$$
\begin{aligned}
0 & =\left.\frac{d}{d t} L\left(\Phi_{t}(u), D \Phi_{t}(u) \cdot v\right)\right|_{t=0} \\
& =D_{1} L(u, v) \cdot Y(u)+D_{2} L(u, v) \cdot D Y(u) \cdot v .
\end{aligned}
$$

Comparing this with the above gives $(d / d t)\{F L(v) \cdot Y\}$ $=0$ and proves the assertion.

Proposition 2.4: Let $L: T B \rightarrow \mathbf{R}$ be a Lagrangian, possibly degenerate and let $Z$ be a Lagrangian vector field for $L$. Suppose $\Phi: B \rightarrow D$ is a diffeomorphism. Set $\tilde{L}: T D \rightarrow \mathbf{R}, \tilde{L}=L \circ T \Phi^{-1}$ where $T \Phi: T B \rightarrow T D$ is the tangent (derivative) of $\Phi$. Then a Lagrangian vector field for $\widetilde{L}$ is given as follows:

$$
\tilde{Z}: T D \rightarrow T^{2} D, \quad \tilde{Z}=T(T \Phi) \circ Z \circ T \Phi^{-1} \equiv(T \Phi)_{*} Z
$$

( $\tilde{Z}$ is the "push-forward" of $Z$ by the diffeomorphism $T \Phi)$.

This is a straightforward check using the definitions.

\section{THE EINSTEIN SYSTEM ON $M$ (NO SHIFT AND NO LAPSE)}

In this section we consider the Einstein system in Gaussian coordinates. Given this coordinate choice, the system is described by a nondegenerate Lagrangian formalism (cf. Refs. 2 and 3 ) which we now globalize using the language of Sec.2. We choose as our configuration space $\mathfrak{T}$, the space of all Riemannian metrics on a fixed compact three-dimensional manifold $M$. Thus $\mathbb{T l}$ is an open convex cone in $S_{2}(M)$, the space of two symmetric covariant tensor fields on $M$. Therefore for $g \in \mathfrak{T C}, T_{g} \mathfrak{T l}=S_{2}(M)$, and we can write

$$
T \mathfrak{N}=\mathfrak{M} \times S_{2}(M) .
$$

We equip $\mathscr{N}$ with an indefinite metric $\mathcal{G}$, referred to as the DeWitt metric, by setting for $g \in \mathfrak{T}$,

$$
\begin{aligned}
& S_{g}: T_{g} \mathfrak{M} \times T_{g} \mathfrak{M} \approx S_{2}(M) \times S_{2}(M) \rightarrow \mathbf{R}, \\
& S_{g}(h, k)=\int_{M}((\operatorname{Tr} h)(\operatorname{Tr} k)-h \cdot k) \mu_{g},
\end{aligned}
$$

where $h \cdot k=h_{i j} k^{i j}$, the dot product of $h$ and $k$, and $\mu_{g}$ $\left(=\sqrt{\operatorname{det} g} d x^{1} \wedge d x^{2} \wedge \cdots \wedge d x^{n}\right)$ is the usual volume element associated with the metric $g$. Note that the space $I T$ has the feature, appropriate for general relativity, of having metric structures which do not depend on any particular Riemannian metric for the underlying manifold $M$. In other classical field theories, such as electrodynamics and hydrodynamics, it is necessary to specify a priori a metric on $M$. In general relativity such a specification is, of course, unnatural as it is precisely the metric structure of $M$ that is evolving.

Proposition 3. 1: The Lagrangian $L_{0}(g, h)=$ $\frac{1}{2} S_{g}(h, h)$ is nondegenerate and the associated Lagrangian vector field exists and is given by

$$
\begin{aligned}
& \mathrm{Z}: \mathfrak{M} \times S_{2}(M) \rightarrow S_{2}(M) \times S_{2}(M) \\
& \begin{aligned}
\mathrm{Z}(g, k) & =\left(k, k \times k-\frac{1}{2}(\operatorname{Tr} k) k+\frac{1}{8}\left[(\operatorname{Tr} k)^{2}-k \cdot k\right] g\right) \\
& \equiv\left(k, S_{g}(k)+\frac{1}{4} \mathfrak{K} g\right),
\end{aligned}
\end{aligned}
$$


where $(k \times k)_{i j}=k_{i l} k_{k}^{l}$, the cross product of $k$ and $k$, $S_{g}(k)=k \times k-\frac{1}{2}(\operatorname{Tr} k) k$, and $K=\frac{1}{2}\left((\operatorname{Tr} k)^{2}-k \cdot k\right)$, the kinetic energy scalar.

For each $(\stackrel{g}{g}, \hat{k}) \in \mathscr{T}\left(\times S_{2}(M)\right.$, there exists a unique smooth curve $\left(g_{t}, k_{t}\right) \in \mathfrak{N} \times S_{2}(M)$ with initial conditions $\left(g_{0}, k_{0}\right)=\left(g, k^{\circ}\right)$ and which satisfies $Z$, for $t$ in some interval about 0 .

Proof: That $S$ is nondegenerate follows from the fact that if $g_{g}(k, h)=0$ for all $h \in S_{2}(M)$, then in particular, setting $h=\frac{1}{2}(\operatorname{Tr} k) g-k, 0=S_{g}\left(k, \frac{1}{2}(\operatorname{Tr} k) g-k\right)$ $=\int_{M} k \cdot k \mu_{g}$, which implies $k=0$ as the integrand is positive.

We first consider Lagrange's equation in the form $(d / d t) D_{2} L_{0}(g, k) \cdot \omega=D_{1} L_{0}(g, k) \cdot \omega, \omega \in S^{2}(M)$. Note that $L_{0}: \mathfrak{M} \times S_{2}(M) \rightarrow \mathbf{R}$, so that $D_{1} L_{0}(g, k)$ : $S_{2}(M) \rightarrow \mathbf{R}$ and $D_{2} L_{0}(g, k): S_{2}(M) \rightarrow \mathbf{R}$. Let $g_{t}$ be a curve in $\mathbb{N}$ that satisfies

$$
g_{0}=g \text { and }\left.\frac{d g}{d t}\right|_{t=0}=\omega \in S_{2}(M)
$$

Then from the rule for taking partial derivatives,

$$
D_{1} L_{0}(g, k) \cdot \omega=\left.\frac{d}{d t} L_{0}\left(g_{t}, k\right)\right|_{t=0}
$$

Similarly,

$$
\begin{aligned}
D_{2} L_{0}(g, k) \cdot \omega=\frac{d}{d t} L_{0}\left(g, k_{t}\right) & \left.\right|_{t=0} \\
& \quad \text { where }\left.\left(k_{t}, \frac{d k_{t}}{d t}\right)\right|_{t=0}=(k, \omega) .
\end{aligned}
$$

By applying this rule, Lagrange's equation becomes

$$
\begin{aligned}
\frac{d}{d t} \int_{M}[(\operatorname{Tr} k) g-k] \cdot \omega \mu_{g}, \\
=\int_{M}\left[-(\operatorname{Tr} k) k+k \times k+\frac{1}{2} \mathcal{K} g\right] \cdot \omega \mu_{g},
\end{aligned}
$$

where we have used the fact that the derivative of the map

$$
\mu: \mathfrak{I T} \rightarrow \Omega(M), \quad g \mapsto \mu_{g}
$$

$[\Omega(M)=$ smooth volume elements on $M]$ is given by

$$
D \mu(g): S_{2}(M) \rightarrow \Lambda^{n}(M), \quad g \mapsto D \mu(g) \cdot \omega=\frac{1}{2}(\operatorname{Tr} \omega) \mu_{g}
$$

$\left[\Lambda^{n}(M)=\right.$ the space of smooth $n$-forms on $\left.M\right]$.

In coordinates this result follows from the formula

$$
\left.\frac{d}{d t} \operatorname{det}\left(g_{i j}+t \omega_{i j}\right)\right|_{t=0}=\operatorname{Tr}\left(\omega_{i j}\right) \operatorname{det}\left(g_{i j}\right)
$$

Since $\omega \in S_{2}(M)$ is arbitrary, the integrands of (3.1) are equal and we get

$$
\frac{d}{d t}[(\operatorname{Tr} k) g-k]^{-1} \mu_{g}=\left[k \times k-(\operatorname{Tr} k) k+\frac{1}{2} \mathcal{K} g\right]^{-1} \mu_{g},
$$

where we are using the superscript -1 to mean that all indices are contravariant, and juxtaposition, like $k^{-1} \mu_{g}$, to mean the tensor product $k^{-1} \mu_{g}=k^{-1} \otimes \mu_{g}$ (classically, expressions like $k^{-1} \mu_{g}$ are tensor densities, written in coordinates as $k^{i j} \sqrt{\operatorname{det} g}$ ).

Equation (3.2) is Lagrange's equation on $T^{*} M$. To get Lagrange's equation on $T \mathscr{T}$, we pull $(3.2)$ back using the fiber derivative of $L_{0}$. This is equivalent to solving (3.2) directly for $d k / d t$. Using the facts that $d g / d t=k, d g^{-1} / d t=-k^{-1}$ (in coordinates this is $\left.d g^{i j} / d t=-g^{i a g j b} k_{a b}\right)$ and $d \mu_{g} / d t=\frac{1}{2}(\operatorname{Tr} k) \mu_{g}$ (in coordinates this is $\left.(d / d t) \sqrt{\operatorname{det} g}=\frac{1}{2} g^{a b}\left(d g_{a b} / d t\right) \sqrt{\operatorname{det} g}\right)$, (3.2) becomes

$$
\begin{aligned}
\left(\frac{d}{d t}[(\operatorname{Tr} k) g-k]^{-1}\right) \mu_{g} & +\frac{1}{2}(\operatorname{Tr} k)[(\operatorname{Tr} k) g-k]^{-1} \mu_{g} \\
& =\left[\left(k \times k-(\operatorname{Tr} k) k+\frac{1}{2} \Re g\right]^{-1} \mu_{g} .\right.
\end{aligned}
$$

Eliminating $\mu_{g}$ gives

$$
\begin{aligned}
\frac{d}{d t}[(\operatorname{Tr} k) g-k]^{-1}=[k & \left.\times k-\frac{1}{2}(\operatorname{Tr} k) k\right]^{-1} \\
& -\frac{1}{2}(\operatorname{Tr} k)^{2} g^{-1}+\frac{1}{2} \mathcal{K} g^{-1} .
\end{aligned}
$$

From $(d / d t)(\operatorname{Tr} k)=-(\operatorname{Tr} k)^{2}+\operatorname{Tr}(d k / d t)$ and taking the trace in (3.3), we find

$$
\frac{d}{d t}(\operatorname{Tr} k)=-\frac{1}{2}(\operatorname{Tr} k)^{2}+\frac{3}{4} \mathfrak{K}
$$

and thus

$$
\frac{d k^{-1}}{d t}=-\left[k \times k-\frac{1}{2}(\operatorname{Tr} k) k\right]^{-1}-(\operatorname{Tr} k) k^{-1}+\frac{1}{4} \mathcal{K} g^{-1} .
$$

Lowering the indices on $k^{-1}$ by using $(d k / d t)^{-1}=$ $\left(d k^{-1} / d t\right)+2(k \times k)^{-1}$ (in coordinates this is $\left.g^{a i} g^{b j}\left(d k_{a b} / d t\right)=\left(d k^{i j} / d t\right)+2 k_{a}^{i} k^{a j}\right)$ gives the result,

$$
\frac{d k}{d t}=k \times k-\frac{1}{2}(\operatorname{Tr} k) k+\frac{1}{4} \mathcal{K} g .
$$

Finally we remark that $Z$ is simply algebraic in $g$ and $k$ and is thus a smooth vector field on $T \mathrm{~T}$ and on $T \mathscr{T}^{k}, 0 \leq k<\infty$, where $\mathscr{T}^{k}$ is the manifold of $C^{k-}$ Riemannian metrics on $M$ in the $C^{k}$ topology (uniform convergence of derivatives up to order $k$ ). The manifolds $T \mathscr{T}^{k}, 0 \leq k<\infty$ are Banach manifolds and hence the usual Picard method for ordinary differential equations is sufficient to establish that $Z$ has a smooth flow on $T_{\mathfrak{T}}{ }^{k}, 0 \leq k<\infty$, defined for a short time interval and a simple limit argument shows that $k=\infty$ is also allowed. Indeed, what one must show is that the time of existence $\epsilon_{k}$ does not go to zero as $k \rightarrow \infty$. To show this, suppose we have a solution $(g, k)$ in $T \mathscr{N}^{k}$ with initial data $\left(g_{0}, k_{0}\right)$ which are $C^{k+1}$. Then we assert the solution is $C^{k^{+1}}$ as long as it is defined in $C^{k}$. This comes from examining the linear differential equation for the spatial derivative of $(g, k)$, as in Refs. 13 and 14. The result then follows.

The second order equation $Z(g, k)=\left(k, Z_{2}(g, k)\right)=$ $\left(k, k \times k-\frac{1}{2}\left[(\operatorname{Tr} k) k+\frac{1}{8}(\operatorname{Tr} k)^{2}-k \cdot k\right] g\right)$ is quadratic in the second or velocity variable $k$; that is, for $\lambda \in \mathbf{R}$, $Z_{2}(g, \lambda k)=\lambda^{2} Z_{2}(g, k)$. A second order equation with this property is called a spray (see Lang12, p. 67). If $Z$ is the spray of a metric it is called a geodesic spray and its base integral curves are the geodesics of the metric. Thus the $Z$ of Proposition 3.1 is the geodesic spray of the DeWitt metric. However, Propositions 3.3 and 3.4 below show that the truncated quantity $S_{g}(k)=k \times k-\frac{1}{2}(\operatorname{Tr} k) k$ also enters the equations in a fundamental way. We will refer to $S_{g}(k)$ as the DeWitt spray.

We remark that the geodesics of $S$ (that is, the base integral curves of $Z$ ) can in fact be found explicitly as has been done by Dewitt ${ }^{3}$ and in a different context by Eardley, Liang, and Sachs. ${ }^{15}$ From these explicit formulas, it is seen that the geodesics exist for short time only, as they eventually run out of $\mathfrak{T}$ into $S_{2}(M)$. 
When this happens, the equations break down and become singular since they involve taking the inverse of $g$.

Since $S$ is nondegenerate, the bundle map $g^{b}: T \mathfrak{N} \rightarrow$ $T^{*} \mathfrak{T}$ defined by $S_{g}^{b}(h) \cdot k=S_{g}(h, k)$ is injective. This map in the classical case $g^{b}: T M \rightarrow T^{*} M$ corresponds to lowering of indices by a metric tensor $g_{i j}$. Note, however, that since the model space $S_{2}(M)$ is the space of covariant tensor fields, $g^{b}(k) \in T_{g}^{*} \Re($ has contravariant indices.

The terminology weakly nondegenerate comes from the fact that the map $S^{b}$, while injective, need not be an isomorphism, cf. Ref. 11. We should also point out that in contrast to strong metrics (as in Ref.12), weak metrics need not have geodesics. For the DeWitt metric, however, the existence of geodesics was checked directly in Proposition 3.1.

Now $S_{g}^{b}(k) \cdot h=g_{g}(k, h)=\int_{M}[(\operatorname{Tr} k)(\operatorname{Tr} h)-k \cdot h] \mu_{g}$ $=\int_{M}[(\operatorname{Tr} k) g-k]^{-1} \cdot h \mu_{g}$. If we consider $S^{2}(\mathrm{M}) \otimes \mu_{g}$ (= space of two-symmetric contravariant tensor densities) as a subspace of $T_{g}^{*} \mathfrak{T}$, the continuous linear functionals on $S_{2}(\mathrm{M})=T_{g} \mathrm{~N}$ in the $C^{\infty}$-topology of uniform convergence of derivatives of all orders, then we can set $S_{g}^{b}(k)=[(\operatorname{Tr} k) g-k]^{-1} \mu_{g}$. The space $T_{g}^{*} \mathfrak{N}$ is a space of tensor-valued distributions.

We will denote by $\pi$ the momentum canonically conjugate to the velocity $k, \pi=g_{g}^{b}(k) \in S^{2}(M) \otimes \mu_{g}$. In coordinates, $\pi^{i j}=\left[\left(g^{a b k_{a b}}\right) g^{i j}-k^{i j}\right] \sqrt{\operatorname{det} g}$ which is the expression for $\pi$ that appears in Ref. 2. Thus $\pi$ can be interpreted as $k$ with its indices raised by the DeWitt metric.

If we consider $S^{2}(M) \otimes \mu_{g}$ as the range of $S_{g}^{b}$ rather than $T_{g}^{*} \Re$, then $g_{g}^{b}$ is onto, and its inverse

$$
\left[S_{g}^{b}\right]^{-1}=S_{g}^{\#}: S^{2}(M) \otimes \mu_{g} \rightarrow S_{2}(M)
$$

is given by

$$
S_{g}^{\#}\left(\pi^{\prime} \otimes \mu_{g}\right)=\frac{1}{2}\left(\operatorname{Tr} \pi^{\prime}\right) g-\left(\pi^{\prime}\right)^{b}\left[=\frac{1}{2}\left(\operatorname{Tr} \pi^{\prime}\right) g_{i j}-\pi_{i j}^{\prime}\right],
$$

where $\pi^{\prime}$ is the tensor part of $\pi=\pi^{\prime} \otimes \mu_{g}$, and $\left(\pi^{\prime}\right)^{b}$ means $\pi^{\prime}$ with its indices lowered $\left[\left(\pi^{\prime}\right)_{i j}^{b}=g_{i k} g_{j l}\left(\pi^{\prime}\right)^{k l}\right.$ $\left.=\pi_{i j}^{\prime}\right]$. The factor $\frac{1}{2}$ enters so that $g_{g}^{\#}\left[g_{g}^{b}(k)\right]=$

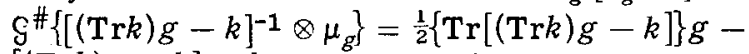
$[(\operatorname{Tr} k) g-k]=k$, as we expect.

We now proceed to consider the gravitational potential of DeWitt and to compute its gradient with respect to the metric \&; see the discussion following Proposition 2.2. Although the spray $Z$ of $\mathrm{S}$ was simply algebraic, the gradient of the potential will be a nonlinear differential operator.

Let

$$
V: \mathfrak{T} \rightarrow \mathbf{R}, g \mapsto 2 \int_{M} R(g) \mu_{g},
$$

where $R(g)$ is the scalar curvature of $g$, and set

$$
L(g, k)=\frac{1}{2} S_{g}(k, k)-V(g) .
$$

Our $k$ and $L$ is minus twice DeWitt's ${ }^{3}$ so that our $L$ is of the form kinetic energy minus potential energy and the kinetic energy enters with the classical factor $\frac{1}{2}$.

Adding this potential to $L_{0}$ in Proposition 3.1 adds a forcing or gradient term to the equations of motion, which we now compute. We include a positive scalar function $N: M \rightarrow \mathbf{R}$ in the potential for later use (see Sec. 8).

Proposition 3.2: Let $N: M \rightarrow \mathbf{R}$ be a smooth positive scalar function on $M$ and let

Then

$$
V: \mathfrak{T} \rightarrow \mathbf{R}, \quad g \mapsto 2 \int_{M} N R(g) \mu_{g} .
$$

$$
-\operatorname{grad} V=-2 N \operatorname{Ric}(g)+\frac{1}{2} N R(g) g+2 \operatorname{Hess} N,
$$

where $\operatorname{Ric}(g)=$ Ricci tensor of $g, \operatorname{Hess} N=N_{|i| j}=$ double covariant derivative, and where

$$
\operatorname{grad} V=S^{\#}(d V): \Im \Pi \rightarrow S_{2}(M)
$$

is computed with respect to the DeWitt metric $\mathcal{G}$.

Proof: We first compute $d V(g)=D V(g): S_{2}(M) \rightarrow \mathbf{R}$. Let $g_{t} \in \mathfrak{T}$ be a curve in $\mathfrak{T}$ with $\left.\left(g_{t}, d g_{t} / d t\right)\right|_{t=0}=$ $(g, \omega) \in \mathscr{T} \times S_{2}$. Then since $d / d t \mu_{g_{t}}=\frac{1}{2} \operatorname{Tr}(d g / d t) \mu_{g_{t}}$, as in the proof of Proposition 3.1, we get

$$
\begin{aligned}
d V(g) \cdot \omega & =\left.\frac{d}{d t} V\left(g_{t}\right)\right|_{t=0} \\
& =\left.2 \int_{M} N \frac{d}{d t}\left(R\left(g_{t}\right) \mu_{g_{t}}\right)\right|_{t=0} \\
& =\left.2 \int_{M}\left[\frac{d R\left(g_{t}\right)}{d t} \mu_{g_{t}}+R\left(g_{t}\right) \frac{1}{2} \operatorname{Tr}\left(\frac{d g_{t}}{d t}\right) \mu_{g_{t}}\right]\right|_{t=0} .
\end{aligned}
$$

A classical computation gives ${ }^{16}$

$$
\left.\frac{d}{d t} R\left(g_{t}\right)\right|_{t=0}=\Delta(\operatorname{Tr} \omega)+\delta \delta \omega-\operatorname{Ric}(g) \omega,
$$

where $\Delta f=-g^{i j} f_{|i| j}=$ Laplace-Beltrami operator on scalars, and where $\delta \delta \omega=\omega^{i j}{ }_{|i| j}=$ the double covariant divergence. Thus

$$
\begin{aligned}
d V(g) \cdot \omega=2 \int_{M} N[\Delta(\operatorname{Tr} \omega)+\delta \delta \omega-\operatorname{Ric}(g) \cdot \omega \\
\left.+R(g) \frac{1}{2}(\operatorname{Tr} \omega)\right] \mu_{g} .
\end{aligned}
$$

Since $M$ is compact without boundary, an integration by parts yields

$$
\begin{aligned}
d V(g) \cdot \omega=2 \int_{M}\{g \Delta N+\operatorname{Hess} N-N[\operatorname{Ric}(g) & \left.\left.-\frac{1}{2} R(g) g\right]\right\} \cdot \omega \mu_{g}
\end{aligned}
$$

and since $\omega$ is arbitrary

$$
d V(g)=2\left\{g \Delta N+\operatorname{Hess} H-N\left[\operatorname{Ric}(g)-\frac{1}{2} R(g) g\right]\right\}^{-1} \mu_{g} .
$$

Using $S_{g}^{\#}$ to pull $D V(g) \in T_{g}^{*} \mathfrak{T}$ back to $T \mathfrak{T}$, we find

$$
\begin{aligned}
& \oint_{g}^{\#}\left[(g \Delta N+\operatorname{Hess} N)^{-1} \mu_{g}\right] \\
& =\frac{1}{2} g \operatorname{Tr}(g \Delta N+\operatorname{Hess} N)-(g \Delta N+\operatorname{Hess} N) \\
& =\frac{1}{2} g(3 \Delta N-\Delta N)-(g \Delta N+\operatorname{Hess} N) \\
& \text { and } \\
& =-\operatorname{Hess} N \\
& \mathrm{~S}_{g}^{\#}\left\{-N\left[\operatorname{Ric}(g)-\frac{1}{2} R(g) g\right]^{-1} \mu_{g}\right\} \\
& =-\frac{1}{2} N g \operatorname{Tr}\left[\operatorname{Ric}(g)-\frac{1}{2} g R(g)\right] \\
& +N\left[\operatorname{Ric}(g)-\frac{1}{2} g R(g)\right]=N\left[\operatorname{Ric}(g)-\frac{1}{4} g R(g)\right] \text {. } \\
& \text { Hence, }-\operatorname{grad} V(g)=-2 N \operatorname{Ric}(g)+\frac{1}{2} N g R(g) \\
& +2 \operatorname{Hess} N \text {. }
\end{aligned}
$$


Combining Propositions 3.1 and 3.2 (and taking $N=1$ ) gives

Proposition 3.3: The vector field equations on $T$ TI associated with $L$ are

$$
\left\{\begin{aligned}
\frac{\partial g}{\partial t}= & k, \\
\frac{\partial k}{\partial t}= & k \times k-\frac{1}{2}(\operatorname{Tr} k) k+\frac{1}{8}\left[(\operatorname{Tr} k)^{2}-k \cdot k\right] g-2 \operatorname{Ric}(g) \\
& +\frac{1}{2} R(g) g \\
= & S_{g}(k)-2 \operatorname{Ric}(g)+\frac{1}{4} \mathcal{H C}(g, k) g,
\end{aligned}\right.
$$

where $\mathcal{H C}(g, k)=\frac{1}{2}\left[(\operatorname{Tr} k)^{2}-k \cdot k\right]+2 R(g)$, and $S_{g}$ is the DeWitt spray.

The basic conservation laws for this system of equations are

Proposition 3.4: Let $\left(g_{t}, k_{t}\right)$ satisfy the equations of Proposition 3. 3:

$$
\left\{\begin{array}{l}
\frac{\partial g}{\partial t}=k, \\
\frac{\partial k}{\partial t}=S_{g}(k)-2 \operatorname{Ric}(g)+\frac{1}{4} \mathcal{F C}(g, k) .
\end{array}\right.
$$

Let $\pi=[(\operatorname{Tr} k) g-k]^{-1} \mu_{g}$. Then

$$
\frac{\partial}{\partial t}(\delta \pi)^{b}=0
$$

and

$$
\frac{\partial}{\partial t}\left[\mathcal{H C}(g, k) \mu_{g}\right]+2 \delta \delta \pi=0,
$$

where $(\delta \pi)^{b}=-\pi_{i}{ }^{j}{ }_{j}$ and

where $\delta \delta \pi=\pi^{i j}{ }_{|i| j}=$ the covariant double divergence. If at $t=0, \delta \pi=0$, then this condition is maintained for all $t$ for which the solution is defined, say $|t|<\epsilon$, and $(\partial / \partial t)\left[\mathcal{H C}(g, k) \mu_{g}\right]=0$. If $\delta \pi=0$ and $\mathcal{H C}(g, k) \equiv 0$ at $t=0$, then $\mathcal{H C}(g, k) \equiv 0$ is maintained for all $t$, $|t|<\epsilon$.

Remark: Note that $(\delta \pi)^{b}$ is conserved whether or not $(\delta \pi)^{b}=0$ initially, but that $\Im c(g, k) \mu_{g}$ is independent of time only if $\delta \pi=0$. It is conceptually best to derive these conservation laws from general symmetry principles. We do that for the divergence condition in Sec. 6 using the symmetry group $\mathscr{D}=\operatorname{Diff}(M)$. In Proposition 8.1 we show that $(\partial / \partial t)\left[\mathcal{H C}(g, k) \mu_{g}\right]=0$ if the theory is invariant under the relativistic timetranslation group $T=C \infty(M ; \mathbf{R})$, which is the case for the empty space field equations. Here we show directly that $(\partial / \partial t)(\delta \pi)^{b}=0$ and $(\partial / \partial t)\left[\mathcal{H C}(g, k) \mu_{g}\right]+2 \delta \delta \pi=0$ for any solution $\left(g_{t}, k_{t}\right)$ of the equations in Proposition 3.4. Of course such a continuity equation is a general feature of Lagrangian field theories. Proposition 3.4, in essence, goes back to Arnowitt-Deser-Misner. ${ }^{2}$

Proof of Proposition 3.4: First we show $(\partial / \partial t)\left[\mathcal{H}(g, k) \mu_{g}\right]+2 \delta \delta \pi=0:$ Let $\mathcal{K}=\frac{1}{2}\left[(\operatorname{Tr} k)^{2}-k \cdot k\right]$ be the kinetic energy scalar. Then from Proposition 3.3 and the proofs of Propositions 3.1 and 3.2 we have

$$
\begin{aligned}
& \frac{d}{d t} \mathcal{K} \mu_{g}= \frac{1}{2} \frac{d k}{d t} \cdot(g \operatorname{Tr} k-k)^{-1} \mu_{g}+\frac{1}{2} k \cdot \frac{d}{d t}\left[(g \operatorname{Tr} k-k)^{-1} \mu_{g}\right] \\
&= \frac{1}{2}\left[k \times k-\frac{1}{2}(\operatorname{Tr} k) k+\frac{1}{4} g \mathcal{K}-2 \operatorname{Ric}(g)\right. \\
&\left.+\frac{1}{2} R(g) g\right] \cdot(g \operatorname{Tr} k-k)^{-1} \mu_{g} \\
&+\frac{1}{2} k \cdot\left\{k \times k-(\operatorname{Tr} k) k+\frac{1}{2} g \mathcal{K}\right. \\
&\left.+2\left[\operatorname{Ric}(g)-\frac{1}{2} R(g) g\right]\right\}^{-1} \\
&= 2\left[\operatorname{Ric}(g)-\frac{1}{2} R(g) g\right] \cdot k . \\
& \text { Also, } \\
& \frac{d}{d t}\left[2 R(g) \mu_{g}\right]=2\left\{\Delta(\operatorname{Tr} k)+\delta \delta k-\left[\operatorname{Ric}(g)-\frac{1}{2} R(g) g\right] \cdot k\right\} \mu_{g}
\end{aligned}
$$

so that

$$
\begin{aligned}
\frac{d}{d t}\left[\mathcal{K} \mu_{g}+2 R(g) \mu_{g}\right] & =2(\Delta \operatorname{Tr} k+\delta \delta k) \mu_{g} \\
& =-2 \delta \delta\left\{((\operatorname{Tr} k) g-k) \mu_{g}\right\} \\
& =-2 \delta \delta \pi .
\end{aligned}
$$

Thus $(\partial / \partial t) \mathcal{H C} \mu_{g}+2 \delta \delta \pi=0$.

To show that $(\partial / \partial t)(\delta \pi)^{b}=0$ we proceed as follows. From Eqs. (3.2) and (3.4) we have

$\frac{\partial \pi}{\partial t}=\left\{k \times k-(\operatorname{Tr} k) k+\frac{1}{2} \pi g+2\left[\operatorname{Ric}(g)-\frac{1}{2} R(g) g\right]\right\}^{-1} \mu_{g} \cdot$

Note that $(3.5)$ is the system of Proposition 3.3 in Hamiltonian form, namely

$$
\frac{d}{d t} D_{2} L(g, k)=D_{1} L(g, k)=-D_{1} H(g, \pi),
$$

where $H(g, \pi)=\frac{1}{2} S_{g}\left(S^{\#}(\pi), S^{\#}(\pi)\right)+V(g)$.

A computation in coordinates shows that

$\frac{\partial}{\partial t}(\delta \pi)^{b}=\left[\delta\left(\frac{\partial \pi}{\partial t}\right)^{b}+\delta\left((\operatorname{Tr} k) k-k \times k-\frac{1}{2} \mathcal{K} g\right) \mu_{g}\right]$.

Substitution of (3.5) into (3.6) yields our result $(\partial / \partial t)(\delta \pi)^{b}=0$.

In the case of electromagnetic fields $E, B$, K $=$ $[g(E, E)+g(B, B)]$ and $-\delta \pi=*(E \wedge B) \mu_{g}$ so that

$$
\begin{aligned}
\frac{d}{d t}\left(\frac{1}{2} \mathcal{H} \mu_{g}\right)+\delta \delta \pi=\frac{d}{d t}\left(\frac{1}{2}[g(E, E)+g(B, B)] \mu_{g}\right) & \\
& -\delta *(E \wedge B)=0 .
\end{aligned}
$$

which is just Poynting's theorem. Here * is the Hodge star operator which maps $k$-forms into $(n-k)$ forms. In this case we do not have $(\delta \pi)^{b}=0$ conserved; cf. Secs. 6, 7, and 8 .

From Propositions 3.3 and 3.4 we find that a solution $\left(g_{t}, k_{t}\right)$ of

$$
\text { (Z) }\left\{\begin{array}{l}
\frac{\partial g}{\partial t}=k, \\
\frac{\partial k}{\partial t}=S_{g}(k)-2 \operatorname{Ric}(g)+\frac{1}{4} \mathcal{H C}(g, k) g,
\end{array}\right.
$$

whose Cauchy data $\left(g_{0}, k_{0}\right)$ at $t=0$ satisfies

(C) $\left\{\begin{aligned} \delta[(\operatorname{Tr} k) g-k] & =0, \\ \mathcal{H}(g, k) & =0,\end{aligned}\right.$ 
satisfies $(C)$ for all time $t$ for which the solution exists, say $|t|<\epsilon$, and hence also satisfies the truncated system of evolution equations

$$
(\bar{E})\left\{\begin{array}{l}
\frac{\partial g}{\partial t}=k, \\
\frac{\partial k}{\partial t}=S_{g}(k)-2 \operatorname{Ric}(g) .
\end{array}\right.
$$

Solutions to the system $(\tilde{E})$ however need not be solutions to $(Z)$, nor is it a priori obvious that solutions to $(\tilde{E})$ preserve the conditions $(C)$ on the Cauchy data. Our next proposition shows, in fact, that the simpler system $(\vec{E})$ is in fact equivalent to $(Z)$ under the hypothesis that the Cauchy data satisfies $(C)$.

Proposition 3.5: Let $\left(g_{t}, k_{t}\right)$ satisfy the system $(\tilde{E})$ with Cauchy data $\left(g_{0}, k_{0}\right)$ that satisfies $(C)$. Then $\nVdash\left(g_{t}, k_{t}\right) \equiv 0$ so that $\left(g_{t}, k_{t}\right)$ also satisfies $(Z)$.

Proof: If $\left(g_{t}, k_{t}\right)$ satisfies $(\tilde{E})$, then a computation as in Proposition 3.4 shows that

$$
\begin{aligned}
\frac{\partial \pi}{\partial t}=\{k \times k-(\operatorname{Tr} k) k+2[\operatorname{Ric}(g) & \left.-\frac{1}{2} R(g) g\right] \\
& -R(g) g\}^{-1} \mu_{g} .
\end{aligned}
$$

Also, a direct verification shows that

$$
\frac{\partial}{\partial t}(\delta \pi)^{b}=\left[\delta\left(\frac{\partial \pi}{\partial t}\right)^{b}+\delta\left((\operatorname{Tr} k) k-k \times k-\frac{1}{2} \mathcal{K} g\right) \mu_{g}\right]
$$

Combining (3.7) and (3.8) gives

$$
\frac{\partial}{\partial t}(\delta \pi)^{b}+\frac{1}{2} \delta\left[\left(\mathcal{H C} \mu_{g}\right) g\right]=0 .
$$

A computation similar to the one in Proposition 3.4 gives

$$
\frac{\partial\left(\mathcal{H} \mu_{g}\right)}{\partial t}+2 \delta \delta \pi+\frac{1}{2}(\operatorname{Tr} k) \mathfrak{H C} \mu_{g}=0 .
$$

Consider (3.9) and (3.10) as a first order linear homogeneous system of partial differential equations for $\left(\mathcal{H C} \mu_{g}\right)$ and $(\delta \pi)^{b}$. Then if $\mathcal{K C} \mu_{g}=0$ and $(\delta \pi)^{b}=0$ at $t=0,(3.9)$ and $(3.10)$ imply $\mathcal{K} \mu_{g}=0,(\delta \pi)^{b}=0$ for all $t$ for which $\left(g_{t}, k_{t}\right)$ satisfies $(E)$. Hence $\left(g_{t}, k_{t}\right)$ satisfies $(Z)$.

We remark that the proposition also follows if we assume that solutions to $(\tilde{E})$ are unique. ${ }^{14}$ Let $\left(g_{t}, k_{t}\right)$ be a solution of $(\tilde{E})$ with Cauchy data that satisfies $(C)$, and let $\left(\bar{g}_{t}, \bar{k}_{t}\right)$ be a solution to $(Z)$ with this same Cauchy data. Then $\left(\bar{g}_{t}, \vec{k}_{t}\right)$ is a solution to $(\tilde{E})$ and by the uniqueness assumption, $\left(g_{t}, k_{t}\right)=\left(\bar{g}_{t}, \bar{k}_{t}\right)$ is also a solution to $(Z)$. Unfortunately, there is not as yet a direct existence or uniqueness proof for the system $(\widetilde{E})$. Using a four-dimensional formulation and a theorem of Leray, Lichnerowicz ${ }^{17}$ proves that Cauchy data

$$
\left(g_{\mu \nu}\left(x^{i}\right), \frac{\partial g_{\mu \nu}}{\partial t}\left(x^{i}\right)\right), \quad 0 \leqslant \mu, \nu \leqslant 3,
$$

of Sobolev class $\left(H^{s}, H^{s-1}\right)$ evolves for short time into a space-time of class $H^{s-1}$. Using an improvement of the Leray theorem by Dionne, Choquet-Bruhat ${ }^{13}$ shows in fact that the space-time is of class $H^{s}$. In a forthcoming paper we give a simple direct proof that $\left(H^{s}, H^{s-1}\right)$ Cauchy data evolves for short time into a space-time of class $H^{s}, s \geqslant 4$ (see Refs. 18 and 19).

Eardley, Liang, and Sachs ${ }^{15}$ give conditions when the Ricci term may be neglected, called velocity-dominated solutions. This condition prevails when the metric is changing very fast as compared with its curvature, as for example near a singular hypersurface. As $\operatorname{Ric}(g)$ is the only term involving spatial derivatives, neglecting Ric $(g)$ reduces the equations to the geodesic equations on $\mathrm{IT}$ which can then be solved explicitly.

As explained in Ref. 1, the evolution equations $(\tilde{E})$ plus the initial conditions $(C)$ are equivalent to the statement that the Lorentz metric $g^{L}$ given in a neighborhood $(-\epsilon, \epsilon) \times M$ of the initial hypersurface $\{0\} \times M$ by

$$
g^{L}(t, m) \cdot\left(\left(r, v_{m}\right),\left(s, w_{m}\right)\right)=g_{t}(m)\left(v_{m}, w_{m}\right)-r s,
$$

where $\left(r, v_{m}\right),\left(s, w_{m}\right) \in T_{(t, m)}(\mathbf{R} \times M) \approx \mathbf{R} \times T_{m} M$ and $g_{t}$ is the time-dependent metric with interval of existence $(-\epsilon, \epsilon)$, is Ricci flat; that is, Einstein's empty space field equations hold. In coordinates the formula reads

$$
g_{\alpha \beta}^{L} d x^{\alpha} d x^{\beta}=-d t^{2}+g_{i j} d x^{i} d x^{j}
$$

where $x^{\alpha}=\left(t, x^{i}\right)$. As we shall explain in Sec. 7 , there are compelling reasons why we want to restrict our solutions to satisfy $(C)$ in addition to the fact that only then do the solutions correspond to Ricci flat space-times.

Note that we are not postulating that the whole spacetime is of the form $\mathbf{R} \times M$; rather it is of the form $(-\epsilon, \epsilon) \times M$ only in a tubular neighborhood of the initial hyperspace. As the metric evolves in time, the topology of the space-time structure could become more intricate. This global aspect in time is a difficult problem, closely related to the singularity problem, about which little is known. $17,19,20$

The above construction is for a space-time in Gaussian coordinates $g_{00}=-1, g_{0 i}=0$. To get the most general space-time, we must modify the equations of evolution to include the shift and lapse function.

We shall deal with the shift and lapse separately since their geometrical meanings are quite different; but they can be handled simultaneously or in succession. When done together, one uses the semidirect product group structure on $T \times D$.

\section{EINSTEIN'S EQUATIONS WITH A SHIFT}

If $g_{0 i}$ is nonzero and we write $X=X^{i}=-g^{i j} g_{0 j}$ ( $\mathrm{g}^{i j}$ is the inverse of the time-dependent 3-metric $g_{i j}$ ) so that $X$ is a time-dependent vector field on $M$, then the evolution equations corresponding to the metric

$g_{\alpha \mathrm{B}} d x^{\alpha} d x^{\beta}=-\left(1-X^{i} X_{i}\right) d t^{2}-2 X_{i} d t d x^{i}+g_{i j} d x^{i} d x^{j}$

are

$$
\left\{\begin{array}{l}
\frac{\partial g}{\partial t}=k-L_{X} g, \\
\frac{\partial k}{\partial t}=S_{g}(k)-2 \operatorname{Ric}(g)-L_{X} k .
\end{array}\right.
$$


Because of the presence of the Lie derivative in the first term, $k$ is no longer the canonical velocity associated with the configuration field $g$, but is now a supplementary variable, defined to simplify the evolution equations; that is, the equation for $\partial k / \partial t$ is simpler than the equation for the acceleration $\partial^{2} g / \partial t^{2}$ $=\partial k / \partial t-(\partial / \partial t)\left(L_{X} g\right)$. We let $h=\partial g / \partial t$ denote the velocity canonically conjugate to the field $g$.

In Ref. 3 the above equations are considered as Lagrange's equations on $\mathbb{M}$. However in this approach the kinetic energy $K(g, h)=\frac{1}{2} g_{g}\left(h+L_{X} g, h+L_{X} g\right)$ fails to be a quadratic function of the velocity $h$ (although it is a quadratic function of the supplementary variable $k$ ).

In order to have a kinetic energy term which is quadratic, and to incorporate the shift vector field into the theory in a natural way, we enlarge the configuration space $\mathfrak{M}$ to $D \times \mathfrak{M}$. We recall that $D=\operatorname{Diff}(M)$ is the group of all smooth orientation-preserving diffeomorphisms of $M$. We can still regard the equations of Proposition 3.3 on $D \times \mathfrak{T}$ by just ignoring the factor D; namely, for $X \in T D,(g, h) \in T \mathfrak{T}$ we have

$$
\begin{cases}\frac{\partial \eta}{\partial t}=X, & \frac{\partial g}{\partial t}=h, \\ \frac{\partial X}{\partial t}=\dot{X}, & \frac{\partial h}{\partial t}=S_{g}(h)-2 \operatorname{Ric}(g)+\frac{1}{4} \mathcal{H C}(g, h) .\end{cases}
$$

These equations come from a degenerate Lagrangian on $D \times M, \bar{L}(X, g, h)=L(g, h)$. The degeneracy is clear because the Lagrangian $\bar{L}: T(D \times \mathfrak{T}) \rightarrow \mathbf{R}$ does not depend on $X$; thus, $X$ can be specified arbitrarily. We give a less trivial extension of the Lagrangian $L$ to $\mathbb{D} \times \mathbb{T}$ shortly.

At this point it will be necessary to set out a few properties of the diffeomorphism group $D$ of our manifold $M$. We shall need only the most elementary aspects of this group, which can all be understood rather easily, as we shall explain. For the more detailed analysis, consult Refs. 7 and 10 and related references.

To begin with, $D$ is an infinite-dimensional manifold. It is not a linear space, as $M$ does not have a linear structure, but $D$ is locally like $C^{\infty}$ functions; hence it is plausible that $D$ has the structure of a manifold modeled on a Fréchet space of $C^{\infty}$ (vector) functions.

What we would like to demonstrate is that the tangent space $T_{\eta} D$ at a point $\eta \in \mathcal{D}$ is the set of smooth maps $X_{\eta}: M \rightarrow T M$ which cover $\eta$; that is, such that the following diagram commutes:

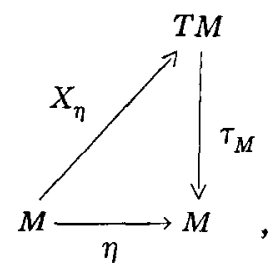

where $\tau_{M}$ denotes the canonical projection of $T M$ to $M$. To see that $X_{\eta}$ is of the form described, let $\eta_{t} \in \mathcal{D}$ be a curve in $D, \eta_{0}=\eta$, so that $\left.\left(d \eta_{t} / d t\right)\right|_{t=0}$ represents a tangent vector in $T_{\eta} D .8$ But for fixed $m \in M, \sigma(t)=\eta_{t}(m)$ is a curve in $M$ with $\sigma(0)=\eta(m)$ and with tangent vector

$$
\sigma^{\prime}(0)=\left.\frac{d \eta_{t}}{d t}(m)\right|_{t=0} \in T_{\eta(m)} M
$$

Thus $d \eta_{t} / d t$ is a map from $M$ to $T M$ covering $\eta$.

We refer to $X_{\eta}$ as a vector field which covers $\eta$, so that $T D$ is the manifold of vector fields covering diffeomorphisms. In particular, $T_{e} D=\Upsilon(M)=\{$ the vector space of smooth vector fields on $M\}=\{$ the Lie algebra of $D\}$. There is a natural projection $\bar{\tau}: T \mathscr{D} \rightarrow \mathscr{D}$ defined by $\bar{\tau}\left(X_{\eta}\right)=\tau_{M} \circ X_{\eta}=\eta \in \mathscr{D}$. For $X_{\eta} \in T_{\eta} \mathfrak{D}, X_{\eta} \circ \eta^{-1}$ is an "ordinary" vector field on $M$. The assertion $d \eta_{t} / d t=X_{\eta_{t}}$ means that $\eta_{t}$ is the flow of the time-dependent vector field $X_{t}=X_{\eta_{t}}{ }^{\circ} \eta_{t}^{-1}=$ $d \eta_{t} / d t \circ \eta_{t}^{-1}$. In other words, $\eta_{t}$ gives the integral of the ordinary differential equations defined by $X_{t}$, or

$$
\frac{d \eta_{t}(x)}{d t}=X_{t}\left(\eta_{t}(x)\right)
$$

Now we introduce a new manifold $Q$ of maps of the form $g \circ \eta$ where $g \in \mathscr{N}$ and $\eta \in \mathcal{D}$. This is isomorphic to $\mathcal{D} \times \mathfrak{T}$ by mapping $(\eta, g) \mapsto g \circ \eta$. This map may be viewed as realizing $C$ as $D \times M$ by right translation or as $a$ in body coordinates. We can also realize $\propto$ in space coordinates using "left translation":

$$
g \circ \eta \mapsto\left(\left(\eta^{-1}\right)^{*} g, \eta\right),
$$

where $\left(\eta^{-1}\right)^{*} g$ represents a new metric obtained by "actively changing the coordinates" by the diffeomorphism $\eta$. If we let $X_{m}, Y_{m} \in T_{m} M$, then

$\left(\eta^{-1}\right)^{*} g(m) \cdot\left(X_{m}, Y_{m}\right)=g \circ \eta^{-1}(m) \cdot\left(T_{m} \eta^{-1}\left(X_{m}\right), T_{m} \eta^{-1}\left(Y_{m}\right)\right)$.

In a coordinate system, $x=\left(x^{1}, \ldots, x^{n}\right)$, this operation $\left(\eta^{-1}\right)^{*} g$ reads as follows: Let $\bar{x}^{i}$ be the $i$ th coordinate of $\bar{x}=\eta(x)$; we suppose for simplicity that the coordinate chart is so large that $\eta$ maps it to itself. Then the new $g$ has coordinates in this system given by

$$
\bar{g}_{i j}(\bar{x})=\frac{\partial x^{k}}{\partial \bar{x}^{i}}(x) \cdot \frac{\partial x^{l}}{\partial \bar{x}^{j}}(x) g_{k l}(x) .
$$

Our conventions on the placing of the stars agrees with the convention in Ref. 12 but is the opposite of that in Ref. 8. For example, $(\eta \circ \zeta)^{*} g=\zeta^{*} \eta^{*} g$.

Our procedure of realizing $Q$ in these two ways as $D \times \mathscr{T}$ is entirely analogous to what occurs in the rigid body and hydrodynamics, cf. Ref. 7 . This is explained further in Sec. 5 below.

We refer to $a$ as the manifold of Riemannian metrics which cover diffeomorphisms, that is, $g_{\eta} \in Q$ covers the diffeomorphism $\eta$ if the following diagram commutes:

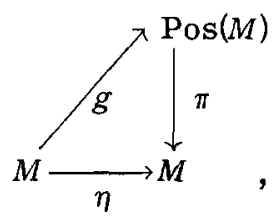

where $\operatorname{Pos}(M)$ is the bundle of positive-definite symmetric two-covariant tensors (not tensor fields) on $M$.

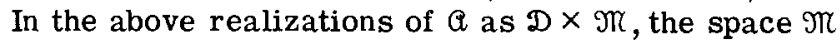
plays the role of the "Lie algebra" of $\propto$, so that right pullback to the "Lie algebra" is given by $g_{\eta} \mapsto g_{\eta} \circ \eta^{-1}$, and left pullback is given by $g_{\eta} \mapsto\left(\eta^{-1}\right)^{*}\left(g_{\eta} \circ \eta^{-1}\right) ; g_{\eta} \circ \eta^{-1}$ is the representation of $g_{\eta}$ in body coordinates, $\left(\eta^{-1}\right)^{*}$ 
$\left(g_{\eta} \circ \eta^{-1}\right)$ is the representation of $g_{\eta}$ in space coordinates, and the transition from body to space coordinates is given by $g_{\eta} \circ \eta^{-1} \mapsto\left(\eta^{-1}\right)^{*}\left(g_{\eta} \circ \eta^{-1}\right)$; that is, this map represents the transition from the "body representation" to the "space representation." This can also be interpreted more mundanely as the transition from a stationary to a moving frame.

To transfer the Lagrangian on $\mathfrak{N}$ to $D \times \mathfrak{K}$, we consider the map

$$
\Phi: \mathfrak{D} \times \mathfrak{T} \rightarrow \mathfrak{K}, \quad(\eta, g) \mapsto \eta^{*} g
$$

( $\Phi$ is the standard left action of the group $D$ on $T$ ).

The tangent (or derivative) of $\Phi$ (not the tangent action) is easily computed. We shall prove that

$$
\begin{aligned}
T \Phi: T D \times T M K \rightarrow & T \mathscr{M} \approx \mathscr{M} \times S_{2}(\mathscr{K}), \\
& \left(X_{\eta},(g, h)\right) \mapsto\left(\eta^{*} g, \eta^{*}\left(h+L_{X_{\eta} \circ \eta^{-1}} g\right)\right) .
\end{aligned}
$$

Note that $X_{\eta} \circ \eta^{-1}$ is an ordinary vector field so that $L_{X_{\eta} \cap \eta-1} g$ is the usual Lie derivative. The proof that $T \Phi$ is as given follows from a lemma from geometry.

Lemma 4.1: If $Y_{t}$ is a time-dependent vector field with flow $\eta_{t}\left(\eta_{0}=i d_{M}=\right.$ identity), then for $g \in \mathfrak{K}$

$$
\frac{d}{d t}\left(\eta_{t}^{*} g\right)=\eta_{t}^{*}\left(L_{Y_{t}} g\right)
$$

This is the usual fundamental theorem connecting flows and Lie derivatives. 8

To prove the formula for $T \Phi$, we may proceed as follows: Let $\eta_{t}$ be the flow of the vector field $X_{\eta} \circ \eta^{-1}$, $\eta_{0}=$ identity, so that as a curve in $D, \eta_{t}$ is tangent to $X_{\eta} \circ \eta^{-1} \circ \eta_{t}$, and $\eta_{t} \circ \eta$ is tangent at $t=0$ to $X_{\eta}$. Let $g_{t}$ be tangent at $t=0$ to $h$ and $g_{0}=g$. Then by definition of the tangent 8

$$
T \Phi\left(X_{\eta},(g, h)\right)=\left.\frac{d}{d t} \Phi\left(\eta_{t} \circ \eta, g_{t}\right)\right|_{t=0} .
$$

Using the definition of $\Phi$ and Lemma 4.1, this becomes

$$
\begin{array}{r}
\frac{d}{d t}\left(\eta_{t} \circ \eta\right)^{*} g_{t=0}=\left.\frac{d}{d t}(\eta)^{*} \eta_{t}^{*} g_{t}\right|_{t=0}=\left.\eta^{*}\left\{\frac{d}{d t} \eta_{t}^{*} g_{t}\right\}\right|_{t=0} \\
=\left.\eta^{*}\left\{\eta_{t}^{*} L_{X_{\eta^{\circ}} \eta^{-1}} g_{t}+\eta_{t}^{*} \frac{d g_{t}}{d t}\right\}\right|_{t=0},
\end{array}
$$

which proves our assertion.

Note: If we had used the right action $\Psi(\eta, g)=$ $\eta_{*} g=\left(\eta^{-1}\right)^{*} g$, the formula for $T \Psi$ would be $(\eta * g$, $\left.\eta_{*} h+L_{x_{\eta^{\circ} \eta^{-1}}}\left(\eta_{*} g\right)\right)$, which is not as convenient for later purposes.

By composing $L$ with $T \Phi$, we can extend our Lagrangian $L: T \Re \rightarrow \mathbf{R}$ to a degenerate Lagrangian $\bar{L}:$ $T(D \times \mathfrak{T}) \rightarrow \mathbf{R}$ given by $\bar{L}=L \circ T \Phi$, that is, $\bar{L}\left(X_{\eta}, g, h\right)$ $=L\left(\eta^{*} g, \eta^{*}\left(h+L_{X_{\eta} \circ \eta^{-1}} g\right)\right)=L\left(g, h+L_{X_{\eta} \circ \eta-1} g\right)$, where the last equality follows from the invariance of $L$ by the pullback action of $D$ (see Sec. 6).

We now explain why the Lagrangian $\bar{L}$ is quadratic in the velocities when viewed as a Lagrangian on $\mathcal{D} \times \mathfrak{K}$, whereas it is not when viewed as a Lagrangian on $T$ IT. We write out $\bar{L}$ as

$\bar{L}\left(X_{\eta}, g, h\right)=\frac{1}{2} S_{g}\left(h+L_{X_{\eta} \circ \eta-1} g, h+L_{X_{\eta}^{\circ} \eta-1} g\right)-2 \int_{M} R \mu_{g}$.
To test whether $\bar{L}$ is quadratic, we must consider the transformation of the velocities $v \mapsto \lambda v, \lambda \in \mathbf{R}$. On $D \times \mathbb{T}$, the velocities are both $X_{n}$ and $h$ (rather than just $h$ ), so that we must consider the transformation $\left(X_{\eta}, h\right) \mapsto\left(\lambda X_{\eta}, \lambda h\right)$. It then follows immediately that

$$
\bar{L}\left(\lambda X_{\eta}, g, \lambda h\right)=\lambda^{2} \bar{L}\left(X_{\eta}, g, h\right)
$$

[whereas $\left.\bar{L}\left(X_{\eta}, g, \lambda h\right) \neq \lambda^{2} \bar{L}\left(X_{\eta}, g, h\right)\right]$. Working on $\mathfrak{T}$ alone, the fact that the shift is also a velocity (but not determined by evolution equations) is obscured; the nonquadratic nature of the Lagrangian on $M$ is trying to tell us that the shift is a velocity variable or equivalently that $D$ should be considered as part of the configuration space.

We also remark that $\bar{L}$ is now a degenerate Lagrangian on $\mathcal{D} \times \mathfrak{M}$, as the metric term is now degenerate. Thus we have achieved a quadratic Lagrangian but only at the expense of giving up a nondegenerate one and also giving up well-defined equations of motion (see Sec. 2). Although it might appear that the price we have had to pay for the exchange is too great, this is not true. The degeneracy leads to an arbitrariness in the evolution equations which allows precisely for an arbitrary specification of a motion of $M$, that is a curve $\eta_{t} \in D$, or its generator, the shift vector field $X_{t}$.

Using $\bar{L}$ and Proposition 2.4, the equations of motion may also be transferred to $D \times \mathfrak{N}$.

Theorem 4.1: Consider on $D \times \mathfrak{N}$ the degenerate Lagrangian

$$
\begin{array}{r}
L(\eta, X, g, h)=\frac{1}{2} S_{g}\left(h+L_{X_{\eta} \circ \eta} g, h+L_{X_{\eta} \circ \eta-1} g\right) \\
-2 \int_{M} R(g) \mu_{g} .
\end{array}
$$

For any curve $\eta_{t}$ with $d \eta_{t} / d t=X_{t} \circ \eta_{t}$, a possible Lagrangian vector field for $L$ is given by the equations

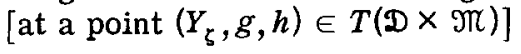

$$
\begin{aligned}
& \left\{\frac{d \zeta_{t}}{d t}=X_{t} \circ \eta_{t}, \quad \frac{\partial g}{\partial t}=k-L_{X} g,\right. \\
& \left\{\frac{d\left(X_{t} \circ \eta_{t}\right)}{d t}=\frac{d Y_{\zeta}}{\partial t}, \quad \frac{\partial k}{\partial t}=S_{g}(k)-2 \operatorname{Ric}(g)+\frac{1}{4} \mathfrak{K}(g, k)\right. \\
& -L_{X} k \text {. }
\end{aligned}
$$

Note: The expression for $L$ may be written (intrisically) directly on the manifold $a$.

As a corollary, we get a simple method for solving the equations with a general shift $X_{t}$ if the solution for $X=0$ is known.

Corollary 4.1: Let $g_{t}, k_{t}$ be a solution of the Einstein system with $N=1, X=0$. Let $X_{t}$ be a given time-dependent vector field with flow $\eta_{t}, \eta_{0}=i d$. Then the solution of the Einstein system with $N=1$ and shift $X_{t}$ and the same initial conditions $\left(g_{0}, k_{0}\right)$ is given by

$$
\bar{g}_{t}=\left(\eta_{t}^{-1}\right)^{*} g_{t}, \quad \bar{k}_{t}=\left(\eta_{t}^{-1}\right)^{*} k_{t} .
$$

To prove the corollary, we need a lemma.

Lemma 4.2: Let $X_{t}$ be a time-dependent vector field and let the flow of $X_{t}$ be $\eta_{t}$. Then $-\left(\eta_{t}^{-1}\right)_{*} X_{t}$ has flow $\eta_{t}^{-1}$, where $\left(\eta_{t}^{-1}\right)_{*} X_{t}=T \eta_{t}^{-1} \circ X_{t} \circ \eta_{t}$ is the "pullback" 
of vector fields $\left[\left(\eta_{t}\right)_{*}\right.$ is "push forward" of vector fields]. Let $g \in \mathfrak{M}$ and $k \in S_{2}(M)$. Then

and

$$
\frac{d}{d t}\left(\eta_{t}^{-1}\right)^{*} g=-L_{X_{t}}\left(\eta_{t}^{-1}\right)^{*} g
$$

$$
\frac{d}{d t}\left(\eta_{t}^{-1}\right)^{*} k=-L_{X_{t}}\left(\eta_{t}^{-1}\right)^{*} k
$$

Proof: Let $X_{t}$ be the generator of $\eta_{t}$ and $Y_{t}$ the generator of $\eta_{t}^{-1}$. By differentiating $\eta_{t} \circ \eta_{t}^{-1}=i d_{M}$, we find

$$
\begin{aligned}
\frac{d}{d t}\left(\eta_{t} \circ \eta_{t}^{-1}\right) & =\frac{d \eta_{t}}{d t} \circ \eta_{t}^{-1}+T \eta_{t} \circ \frac{d \eta_{t}^{-1}}{d t}=X_{t}+T \eta_{t} \circ Y_{t} \circ \eta_{t}^{-1} \\
& =X_{t}+\left(\eta_{t}\right)_{*} Y_{t}=0,
\end{aligned}
$$

where $\left(\eta_{t}\right)_{*} Y_{t}=T \eta_{t} \circ Y_{t} \circ \eta_{t}^{-1}$. Thus $Y_{t}=-\left(\eta_{t}^{-1}\right)_{*} X_{t}$ is the generator of the flow $\eta_{t}^{-1}$. From Lemma 4.1, for $g \in \mathfrak{M}$, or $k \in S_{2}(M)$,

$\frac{d}{d t}\left(\eta_{t}^{-1}\right)^{*} g=\left(\eta_{t}^{-1}\right)^{*} L_{Y_{t}} g=L_{\left(\eta_{t}\right)_{*} Y_{t}}\left(\eta_{t}^{-1}\right)^{*} g=-L_{X_{t}}\left(\eta_{t}^{-1}\right)^{*} g$,

where the second equality follows from the fact that $L_{Y} g$ is a tensor and hence commutes with push forward in each of its arguments; in coordinates this is just covariance with respect to coordinate transformations.

Note: We have given these details because care is required when working with time-dependent vector fields $X_{t}$. Indeed $X_{t}$ is not invariant under its own flow.

Proof of Corollary 4.1: By the lemma we get

$$
\frac{\partial \bar{g}_{t}}{\partial t}=\left(\eta_{t}^{-1}\right)^{*} \frac{\partial g_{t}}{\partial t}-L_{X_{t}} \bar{g}_{t}=\bar{k}_{t}-L_{X_{t}} \bar{g}_{t},
$$

since $\partial g_{t} / \partial t=k_{t}$ when $X_{t}=0$.

Similarly,

$$
\begin{aligned}
\frac{\partial \bar{k}_{t}}{\partial t} & =\left(\eta_{t}^{-1}\right)^{*} \frac{\partial k_{t}}{\partial t}-L_{X_{t}} \bar{k}_{t} \\
& =\left(\eta_{t}^{-1}\right)^{*}\left(S_{g_{t}}\left(k_{t}\right)\right)-2\left(\eta_{t}^{-1}\right)^{*}\left(\operatorname{Ric}\left(g_{t}\right)\right)-L_{X_{t}} \vec{k}_{t} \\
& =S_{\bar{g}_{t}}\left(\vec{k}_{t}\right)-2 \operatorname{Ric}\left(\bar{g}_{t}\right)-L_{X_{t}} \bar{k}_{t},
\end{aligned}
$$

where the last equality again follows from the fact that $S(\cdot, \cdot)$ and $\operatorname{Ric}(\cdot)$ are tensor operators and hence commute with push forward (again, in coordinates, this is just covariance with respect to coordinate transformations), and we have used the equations for $\partial k_{t} / \partial t$ when $X_{t}=0$.

Corollary 4.1 shows that even though the evolution equations with a shift involve extra nonlinear, second order terms [since $L_{X} k=L_{X}\left(h+L_{X} g\right)$ is quadratic in the velocities], the more general system can be solved merely by solving an ordinary differential equation; that is, by finding the flow of $X_{t}$.

The above geometry also makes it transparent how the space-time in the presence of a shift is to be constructed. Namely we have a diffeomorphism

$$
\Psi: \mathbf{R} \times M \rightarrow \mathbf{R} \times M, \quad \Psi(t, m)=\left(t, \eta_{t}(m)\right),
$$

which rotates the space $M$. It transforms, by Corollary 4.1 , the old solution to the new solution. Thus it transforms the old space-time to the new one. It is easy to check that the new space-time metric is the one stated at the beginning of this section. Thus the space-time with a shift is isometric to the spacetime without a shift.

We now explain why we have changed the sign of the shift (see also the next section). If the first evolution equation, for example, were $\partial g_{t} / \partial t=k_{t}+L_{X_{t}} g_{t}$, then we would consider $\bar{g}_{t}=\eta_{t}^{*} g_{t}$ and $\bar{k}_{t}=\eta_{t}^{*} k_{t}$ as the solution with shift $X_{t}$ if $\left(g_{t}, k_{t}\right)$ is the solution with shift zero. But then

$$
\begin{aligned}
\frac{\partial \bar{g}_{t}}{\partial t} & =\eta_{t}^{*} \frac{\partial g_{t}}{d t}+\eta_{t}^{*} L_{X_{t}} g_{t}=\bar{k}_{t}+L_{(\eta \bar{t})_{*} X_{t}}\left(\eta_{t}^{*} g_{t}\right) \\
& =\bar{k}_{t}+L_{\left(\eta \bar{t}^{-}\right)_{*} X_{t}} \bar{g}_{t},
\end{aligned}
$$

so that now the equations depend on $\eta_{t}$ explicitly, which is not natural.

\section{THE EINSTEIN SYSTEM IN SPACE AND BODY COORDINATES}

Interestingly enough, it is possible to interpret the Einstein system (E) in terms analogous to the concepts of space and body coordinates used to describe the motion of a rigid body or of a fluid in hydrodynamics. The basis for this interpretation is the two identifications of $Q$, the manifold of Riemannian metrics which cover diffeomorphisms, with $D \times \mathfrak{N}$; the right identification $g_{\eta} \mapsto\left(\eta, g_{7} \circ \eta^{-1}\right) \in D \times$ Tा leading to what we loosely call "body coordinates," and the left identification $g_{\eta} \mapsto\left(\eta,\left(\eta^{-1}\right)^{*}\left(g_{\eta} \circ \eta^{-1}\right)\right) \in D \times \mathfrak{D}$ leading to "space coordinates." Here $\mathbb{M}$ plays the role of the "Lie algebra" of $Q$ and is therefore analogous to the velocity phase space $T_{e} S O(3) \approx \mathbf{R}^{3}$ for the rigid body or $T_{i d} \mathscr{D}_{\mu}=\mathscr{X}_{0}(M)$ (the space of all divergence free vector fíelds) for hydrodynamics (see Ref. 7).

If we transpose the Lie derivative terms in $(E)$ to the left-hand side, we see that the operator $(\partial / \partial t)+L_{X_{t}}$ enters in the evolution equations for both $g_{t}$ and $k_{t}$. The derivatives $\left(\partial g_{t} / \partial t\right)+L_{X} g_{t}$ and $\left(\partial k_{t} / \partial t\right)+L_{X} k_{t}$ are entirely analogous to the material or Eulerian derivative $\left(\partial X_{t} / \partial t\right)+\nabla_{X} X_{t}$ which appears in hydrodynamics or the time derivative $(d(I \cdot \omega) / d t)+\omega \times(I \cdot \omega)$ of the angular momentum $L=I \cdot \omega$ of a rigid body as observed in space coordinates.

The Eulerian derivative is the total time derivative of the fluid velocity as the fluid moves around in space. Although it is the time derivative of the velocity with respect to an observer who is moving with the fluid, it is expressed in terms of quantities referring to points fixed in space; that is, it is the total time derivative of the fluid as seen by an observer fixed in space. We say that an observer moving with the fluid is in body coordinates, or is "on" the fluid, and an observer fixed in space is in space coordinates, or is "off" the fluid.

We now wish to investigate further this analogy of general relativity with hydrodynamics in which the derivatives $\left(\partial g_{t} / \partial t\right)+L_{x_{t}} g_{t}$ and $\left(\partial k_{t} / \partial t\right)+L_{x} k_{t}$ can be interpreted as the total derivative of a time-dependent metric field $g_{t}$ (or of $k_{t}$ ) as seen by an observer in space coordinates. We let the manifold $M$ be the body. We consider a curve $\eta_{t} \in \mathbb{D}(M), \eta_{0}=i d_{M}$, as describing a rotation of the body $M$. Thus we consi- 
der the points of the manifold $M$ to be moving according to the rule that a point which at time $t=0$ is at $m \in M$ is at $\eta_{t}(m)$ after time $t$. We then make the convention that an observer is in body coordinates if he is on the manifold, and is in space coordinates if he is off the manifold. An observer in body coordinates, as he moves with the manifold, detects no motion of the manifold.

Now let $g_{t}$ be a time-dependent metric field on $M$. We assume that this field is rigidly attached to the body $M$ as it moves according to the curve $\eta_{t}$, so that we set $g_{t}=g_{\text {body }}$ (time-dependence implied). An observer in body coordinates (who detects no motion of $M$ ) then finds $\left(\partial g_{\text {body }} / \partial t\right)=k_{\text {body }}$ as the "velocity" of the metric.

An observer in space coordinates (who is off the manifold) sees the metric field gody as it is dragged past him by the moving manifold. He sees the (timedependent) metric field $g_{\text {space }}=\left(\eta_{t}^{-1}\right)^{*} g_{\text {body }}$ and computes as the "velocity" of $g_{\text {s pace; }}$;

$$
\frac{\partial g_{\text {space }}}{\partial t}=k_{\text {space }}-L_{X} g_{\text {space }}
$$

where $k_{\text {space }}=\left(\eta_{t}^{-1}\right)^{*} k_{\text {body }}=\left(\eta_{t}^{-1}\right)^{*}\left(\partial g_{\mathrm{body}} / \partial t\right)$ and $X$ is the shift vector field which generates the motion $\eta_{t}$ of $M$. Similarly he computes

$$
\frac{\partial k_{\text {space }}}{\partial t}=S_{g_{\text {space }}}\left(k_{\text {space }}\right)-2 \operatorname{Ric}\left(g_{\text {space }}\right)-L_{X} k_{\text {space }} .
$$

But (5.1) and (5.2) are just the evolution equations of (E) with lapse $N=1$.

\section{CONSERVATION OF THE DIVERGENCE CONDI- TION}

In Proposition 3.4 we saw that the divergence condition is maintained by the Einstein equations. Now we want to give a more natural geometric proof of this fact using general symmetry methods.

The idea is extremely simple and goes as follows. Group $D$ acts on $\mathfrak{T}$ by $g \mapsto\left(\eta^{-1}\right)^{*} g$ as we have seen before. We assert that this action is a symmetry for our Lagrangian $L(g, h)=\frac{1}{2} \mathcal{G}_{g}(h, h)-V(g)$ and that the corresponding conserved quantities, computed according to Proposition 2. 3 give us the desired conservation law.

Of course by Corollary 4.1 it is enough to show this for $X=0$; we get the corresponding result with a shift immediately (and for a lapse too using the results below).

Let us denote for fixed $\eta \in \mathcal{D}$, the map $g \mapsto\left(\eta^{-1}\right)^{*} g$ by $\theta_{\eta}$. First we assert that $\theta_{\eta}$ is an isometry for the DeWitt metric. This is almost obvious since $S_{g}$ is defined intrinsically and everything transforms properly (see Refs. 5 or 10 for an analogous result). Secondly, $\theta_{\eta}$ leaves invariant the potential. Indeed, since $R$ is a tensor,

$$
\begin{aligned}
\int_{M} R\left(\left(\eta^{-1}\right)^{*} g\right) \mu_{(\eta-1)}{ }^{*} g & =\int_{M}\left[R(g) \circ \eta^{-1}\right]\left(\eta^{-1}\right)^{*} \mu_{g} \\
& =\int_{M} R(g) \mu_{g}
\end{aligned}
$$

by the change of variables formula $\left[\left(\eta^{-1}\right)^{*} \mu_{g}\right.$ is just the Jacobian of $\eta^{-1}$ times $\mu_{g}$.
We can therefore compute the conserved quantities using Proposition 2.3. Let $\zeta_{t}$ be a one-parameter group of diffeomorphisms generated by a vector field $Z$ on $M$. Then $\theta_{\zeta_{t}}$ is a one-parameter group of motions of $\mathfrak{T}$ leaving $L$ fixed and $\theta_{\zeta_{t}}$ is generated by $g \mapsto-L_{Z} g$, a Killing vector field on $\mathfrak{M}$. The corresponding conserved quantity is therefore the realvalued function on $T$ TM given by

$$
(g, k) \mapsto g_{g}\left(k,-L_{z} g\right)
$$

Lemma 6.1: $\int_{M}\left(L_{Z} g \cdot k\right) \mu_{g}=2 \int_{M}(Z \cdot \delta k) \mu_{g}$.

Proof: As was stated in the introduction, and as is easy to check, we have the formula

$$
L_{Z} g=Z_{i \mid j}+Z_{j \mid i}
$$

From this we see that $\delta(k \cdot Z)=(\delta k) \cdot Z-k \cdot \nabla Z=$ $(\delta k) \cdot Z-\frac{1}{2} k \cdot L_{Z} g$.

By Stokes theorem we have that $\int_{M} \delta(k \cdot Z) \mu_{g}=0$, and so the lemma follows. Recall that $\delta k=-k_{i} j_{1 j}$. Now $S_{g}\left(k, L_{Z} g\right)=\int_{M}\left(L_{Z} g \cdot \pi^{\prime}\right) \mu_{g}$, where $\pi^{\prime}=[(\operatorname{Tr} k) \mathrm{g}-$ $k]^{-1}$ is the tensor part of $\pi=\pi^{\prime} \otimes \mu_{g}$. From

Lemma 6.1 we get the fact that

$$
\int_{M}\left(Z \cdot \delta \pi^{\prime}\right) \mu_{g} \text { is conserved. }
$$

Since $Z$ is arbitrary, $\left(\delta \pi^{\prime}\right)^{b} \otimes \mu_{g}=(\delta \pi)^{b}$ is conserved, where, as above, the symbol ()$^{b}$ indicates that the index is lowered by the time-dependent metric $g_{i j}$, that is $\left(\delta \pi^{\prime}\right)^{b}=-\left(\pi^{\prime}\right)_{i} j_{\mid j}$. Thus if $\delta[(\operatorname{Tr} k) g-k] \stackrel{0}{=} 0$ at $t=0$ then this condition is maintained in time, thereby proving our conservation law.

Note that $\delta \pi^{\prime}$ must be taken with its index down, that is, regarded as a one form, in order that its contration with $Z$ does not involve the metric. Note that we have shown $(\delta \pi)^{b}$ is conserved even if $(\delta \pi)^{b}$ is not zero at $t=0$, although this is not true for $\delta((\operatorname{Tr} k) g$ $-k$ ) because of the $\mu_{g}$ term (see also Ref. 19). This is for the full set of Hamiltonian equations as in Proposition 3.3. For the truncated system it is necessary to require that $\delta \pi$ and $\mathcal{H}$ both be zero at $t=0$ as we saw in Proposition 3.4.

Geometrically, $\delta((\operatorname{Tr} k) g-k)=0$ on $\mathfrak{M}$ means that $k$ is perpendicular to the orbit of $D$ through $g$ (perpendicular in the DeWitt metric). This is exactly a restatement of the condition $\oint_{g}\left(k, L_{Z} g\right)=0$ for all $Z$.

Thus the conservation of $\delta((\operatorname{Tr} k) g-k)$ means that if $(g, k)$ starts off perpendicular to the orbit through $g$, it must evolve in such a manner that it remains perpendicular to the orbit. If a shift is present, we measure perpendicularity by the DeWitt metric on $\mathfrak{D} \times \mathfrak{M}$. In the metric $g_{g}(\cdot, \cdot)$, this means that $g_{t}$, rather than proceeding perpendicular to the orbits, also "slides along" the orbits; this sliding is determined by the flow of the shift vector field.

We also remark that

$$
\begin{aligned}
L_{X} \pi & =L_{X}\left(\pi^{\prime} \otimes \mu_{g}\right)=L_{X} \pi^{\prime} \otimes \mu_{g}+\pi^{\prime} \otimes L_{X} \mu_{g} \\
& =L_{X} \pi^{\prime} \otimes \mu_{g}+(\operatorname{div} X) \pi^{\prime} \otimes \mu_{g},
\end{aligned}
$$

where $L_{X} \mu_{g}=(\operatorname{div} X) \mu_{g}, \operatorname{div} X=-\delta X=X^{i}{ }_{i}$. Thus the Lie derivative of a tensor density has the extra 
divergence term $(\operatorname{div} X) \pi^{\prime}$ in it. Comparing this with the evolution equations in Arnowitt, Deser, and Misner ${ }^{2}$ reveals the last three terms in the equation for $\partial \pi / \partial t$ as $L_{X} \pi$.

In summary, we have proven:

Theorem 6.1: Let $L$ be a Lagrangian system $L(g, k)=\frac{1}{2} S_{g}(k, k)-V(g)$ on TNT with Lagrangian vector field $Z$. Suppose $V$ is invariant under the action of $D$ on $\mathbb{T}$. Then if $(g, k)$ is an integral curve of $Z, \partial / \partial t\left\{\delta[(\operatorname{Tr} k) g-k] \mu_{g}\right\}=0$.

Thus if $\delta[(\operatorname{Tr} k) g-k]=0$ holds at $t=0$, it holds for all time.

In other words, the divergence condition results precisely from the spatial coordinate invariance of our Lagrangian. One can similarly work out laws for other coordinate invariant theories which are built on tensor or vector bundles other than $S_{2}(M)$.

\section{THE EINSTEIN EQUATIONS WITH A LAPSE}

We have just seen that the shift vector field $X$ has a simple geometric interpretation and the solutions to the modified Einstein equations are related to those with zero shift in a very simple and geometrically transparent way. The lapse function is more interesting and a bit more intricate. In dealing with the lapse we may assume the shift is zero.

If one has a Lagrangian function on $T B$, there is a standard way (see Ref. 9, p. 133) of associating a homogeneous Lagrangian on $T(\mathbf{R} \times B)$. Namely set

$$
\begin{aligned}
& \bar{L}: T \mathbf{R} \times T B \approx \mathbf{R} \times \mathbf{R} \times T B \rightarrow \mathbf{R}, \\
& \bar{L}(t, \lambda, v)=\lambda L(v / \lambda), \lambda \neq 0 .
\end{aligned}
$$

Here $\lambda \in T_{t} \mathbf{R}$ is now a velocity, and so $\bar{L}$ is homogeneous of first order in the velocities. $L$ is defined just on the subset where $\lambda \neq 0$. This $\bar{L}$ is degenerate and its base integral curves are obtained from those of $L$ by suspending them in $\mathbf{R} \times B$ with an arbitrary change of parametrization (reflecting the degeneracy). Physically, the time has changed roles from being the evolution parameter of the system to a coordinate in the extended configuration space; one is then free to choose an arbitrary evolution parameter for the system.

With this classical example in mind, we thus extend our Lagrangian $L$ on $T \mathscr{T}, L(g, h)=\frac{1}{2} S_{g}(h, h)-$ $2 \int_{M} \mathrm{R}(g) \mu_{g}$ to $\overleftarrow{L}: T(\mathcal{T} \times \mathfrak{M}) \rightarrow \mathrm{R}$ by setting for $(\xi, N) \in \mathcal{T} \times \mathcal{T}, N>0$,

$$
\begin{aligned}
\bar{L}(\xi, N, g, h) & =\int_{M} N \mathcal{L}\left(g, \frac{h}{N}\right) \mu_{g} \\
& =\frac{1}{2} \int_{M} N\left(\left(\operatorname{Tr} \frac{h}{N}\right)^{2}-\frac{h}{N} \cdot \frac{h}{N}\right) \mu_{g}-2 \int_{M} N R(g) \mu_{g} .
\end{aligned}
$$

Recall $\mathcal{T}$ is the space of smooth functions $\xi: M \rightarrow \mathrm{R}$, $\tau=C^{\infty}(M ; R)$. Since $\mathcal{T}$ is a linear space, $T \mathcal{T}=\mathcal{T} \times \mathcal{T}$ and we denote elements in the tangent space by $(\xi, N) \in \mathcal{T} \times \mathcal{T}$. Note that the constant functions form a subgroup of $\mathcal{T}$ naturally isomorphic to $\mathbf{R}$; restricting to these functions we recover the classical extension of $L$. For the relativistic case, the introduction of $\tau$ instead of $\mathbf{R}$ is quite natural as it allows for observers at different points of $M$ to have different clock rates.
For the classical extension of $L$ to $T(R \times B)$, there is no problem about the existence of its Lagrangian vector field. One can easily check that the most general such second order vector field is given by

$$
\bar{Z}(t, \lambda, v)=\alpha(t, \lambda) \oplus \lambda Z(v),
$$

where $\alpha$ is any second order equation on $T R$ (cf. Ref. 8, p. 136) and $Z$ is the Lagrangian vector field for $L$ on $T B$.

Now we come to a somewhat surprising result. This is that, when we extend $L$ to $T(T \times \mathscr{T})$ as above, the Lagrangian vector field need not exist at every point of $T\left(\mathcal{T}^{\prime} \times \mathfrak{N}\right)$. In fact, in the next theorem we shall see that we are forced to restrict to the set on which $\mathcal{H}$ is identically constant. The result is quite general for any Lagrangian system, although we deal explicitly with the case at hand. This provides the explanation of why $\mathcal{H}$ must be identically constant (generally taken zero) rather than just the total integrated energy being conserved; cf. Misner. ${ }^{4,2}$

Sachs 15 has pointed out that in some dust models, IC can be a nonzero constant. Observe that $\mathcal{H}$ is the total Hamiltonian governing the evolution of all quantities in the theory. For instance in the presence of an electromagnetic field, $J \mathrm{C}=-G_{0}{ }^{0}+T_{0}{ }^{0}$ is the energy governing the evolution of both the gravitational and electromagnetic fields. For a physical solution $\mathcal{H C}=0$; the hypothesis (ii) in the theorem below means physically that our system is relativistic in the sense that one cannot physically distinguish between the various spacelike hypersurfaces. If there are given $a$ priori sources or other "painted on" external fields present, such as the velocity field of a fluid or an electromagnetic field, one can physically distinguish the various hypersurfaces and the hypothesis (ii) will not hold.

Theorem 7.1: (i) If a Lagrangian vector field $\bar{Z}$ for $\bar{L}$ [defined on $T(\mathcal{T} \times \mathfrak{T})$ above] exists, then it must be a second-order equation provided that it is second order in either $\xi$ or $g$. (ii) In order that $\bar{Z}$ should exist as a second-order equation at $(\xi, N, g, h)$ and that $N$ be arbitrarily specifiable, that is, that the "degenerate direction" is all of $T$, it is necessary that for any curve $(\xi(t), N(t), g(t), h(t))$ tangent to $\bar{Z}$, we have $(\partial / \partial t)\left\{\mathcal{H C}(g, h / N) \mu_{g}\right\}=0$, where $\mathcal{H C}(g, h / N)$ $=\frac{1}{2}\left[(\operatorname{Tr}(h / N))^{2}-(h / N) \cdot(h / N)\right]+2 \mathrm{R}(g)$.

Proof: (i) In general the relation between $Z$ and $L$ is the Lagrangian condition

$$
2 \omega_{L}(v)[Z(v), w]=d E(v) \cdot w
$$

on $T B$ (see Sec. 2). If we let $Z=\left(Z_{1}, Z_{2}\right)$ locally on $T B$, this condition reads as follows: For all $e_{1}, e_{2}$ we have

$$
\begin{aligned}
& D_{1} D_{2} L(u, e) \cdot e_{1} \cdot e-D_{1} L(u, e) \cdot e_{1}+D_{2} D_{2} L(u, e) \cdot e_{2} \cdot e \\
&= D_{2} D_{2} L(u, e) \cdot e_{1} \cdot Z_{1}-D_{1} D_{2} L(u, e) \cdot Z_{1} \cdot e_{1} \\
&+D_{2} D_{2} L(u, e) \cdot Z_{1} \cdot e_{2}-D_{2} D_{2} L(u, e) \cdot Z_{2} \cdot e_{1} .
\end{aligned}
$$

These split up into two conditions: and

$$
D_{2} D_{2} L(u, e) \cdot Z_{1} \cdot e_{2}=D_{2} D_{2} L(u, e) \cdot e_{2} \cdot e
$$




$$
\begin{aligned}
& D_{1} D_{2} L(u, e) \cdot e_{1} \cdot e-D_{1} L(u, e) \cdot e_{1} \\
&= D_{1} D_{2} L(u, e) \cdot e_{1} \cdot Z_{1}-D_{1} D_{2} L(u, e) \cdot Z_{1} \cdot e_{1} \\
&-D_{2} D_{2} L(u, e) \cdot Z_{2} \cdot e_{1}
\end{aligned}
$$

In general, we cannot conclude from (7.1) that $Z_{1}(u, e)=e$ because $L$ is degenerate.

Now let us turn to the case at hand. Let us incorporate $\mu_{g}$ into $\mathscr{L}$ so we can briefly just write

$$
\bar{L}(\xi, g, N, h)=\int N \mathscr{L}(g, h / N)
$$

We also suppress the fact that $\mathscr{L}$ depends explicitly on $D g, D^{2} g$, which is irrelevant for the present discussion.

Using obvious notation, the derivatives of $\bar{L}$ are easily worked out to be the following:

$$
\begin{aligned}
& D_{1} L(\xi, g, N, h) \cdot(\xi, g) \\
& =\text { derivative of } L \text { with respect to }(\xi, g) \text { in direc- } \\
& \text { tion }(\tilde{\xi}, \tilde{g}) \\
& =\int N \partial_{g} \mathfrak{L}(g, h / N) \cdot \tilde{g} \text {; } \\
& D_{2} L(\xi, g, N, h) \cdot(\tilde{N}, \tilde{h}) \\
& =\text { derivative with respect to the velocity variables } \\
& (N, h) \text { in direction }(\tilde{N}, \tilde{h}) \\
& =\int \tilde{N} \mathcal{L}\left(g, \frac{h}{N}\right)-\int \tilde{N} \partial_{h} \mathcal{L}\left(g, \frac{h}{N}\right) \cdot \frac{h}{N}+\int \partial_{h} \mathcal{L}\left(g, \frac{h}{N}\right) \cdot \tilde{h} ; \\
& D_{1} D_{2} L(\xi, g, N, h) \cdot(\xi, \tilde{g}) \cdot(\tilde{N}, \tilde{h}) \\
& =\int \tilde{N} \tilde{\partial}_{g} \mathcal{L}\left(g, \frac{h}{N}\right) \cdot \tilde{g}-\int \tilde{N}_{g} \partial_{h} \mathcal{L}\left(g, \frac{h}{N}\right) \cdot \tilde{g} \cdot \frac{h}{N} \\
& +\int \partial_{g} \partial_{h} \mathcal{L}\left(g, \frac{h}{N}\right) \cdot \tilde{g} \cdot \tilde{h} \\
& D_{2} D_{2} L(\xi, g, N, h) \cdot(\tilde{N}, \tilde{h}) \cdot(\tilde{N}, \tilde{\tilde{h}}) \\
& =\int \tilde{N} \tilde{N} \partial 2 \frac{h}{h} \mathcal{L}\left(g, \frac{h}{N}\right) \cdot \frac{h}{N} \cdot \frac{h}{N^{2}}-\int \tilde{\tilde{N}} \partial 2 \mathcal{h}\left(g, \frac{h}{N}\right) \cdot \tilde{h} \cdot \frac{h}{N^{2}} \\
& -\int \tilde{N}_{\delta}^{2} \mathcal{L}\left(g, \frac{h}{N}\right) \cdot \frac{\tilde{h}}{N} \cdot \frac{h}{N}+\int \partial_{h}^{2} \mathcal{L}\left(g, \frac{h}{N}\right) \cdot \tilde{h} \cdot \frac{\tilde{h}}{N} \cdot
\end{aligned}
$$

Note that in the computation of the second derivative of $L$ with respect to the velocity variables, two pairs of terms canceled out. Now let us use this expression to write out condition (7.1). Let us write $Z_{1}(\xi, g, N, h)=(\dot{\xi}, \dot{g})$ for convenience. Condition (7.1) splits into two conditions, taking respectively $e_{2}=(\tilde{N}, 0)$ and $e_{2}=(0, \tilde{h})$. We get

$0=\int \tilde{N} \dot{\xi} \partial_{h}^{2} \mathcal{L}\left(g, \frac{h}{N}\right) \cdot \frac{h}{N} \cdot \frac{h}{N^{2}}-\int \tilde{N} \partial_{h}^{2} \mathcal{L}\left(g, \frac{h}{N}\right) \cdot \frac{\dot{g}}{N} \cdot \frac{h}{N}$

and

$0=-\int \dot{\xi} \partial_{h} \mathcal{L}\left(g, \frac{h}{N}\right) \cdot \tilde{h} \cdot \frac{h}{N^{2}}+\int \partial_{h}^{2} \mathcal{L}\left(g, \frac{h}{N}\right) \cdot \tilde{h} \cdot \frac{g}{N}$.

Each of these conditions is equivalent to the single condition $\dot{\xi} h=N \dot{g}$. Thus if $\dot{\xi}=N$, then $h=\dot{g}$ and vice versa. Hence (i) follows.

To establish (ii), we write out condition (7.2) which now becomes
$D_{1} L(u, e) \cdot e_{1}=D_{1} D_{2} L(u, e) \cdot e \cdot e_{1}+D_{2} D_{2} L(u, e) \cdot Z_{2} \cdot e_{1} \cdot$

Again we have a split into two separate conditions taking, respectively, $e_{1}=(\tilde{N}, 0)$ and $e_{1}=(0, \tilde{h})$. By letting $Z_{2}=(\dot{N}, \dot{h})$ we get

$$
\begin{aligned}
0=\int \tilde{N} \partial_{g} & \left(g, \frac{h}{N}\right) \cdot h-\int \tilde{N} \partial_{g} \partial_{h} \mathcal{L}\left(g, \frac{h}{N}\right) \cdot h \cdot \frac{h}{\bar{N}} \\
& +\int \tilde{N} \dot{N} \partial_{h}^{2} \mathcal{L}\left(g, \frac{h}{N}\right) \cdot \frac{h}{N} \cdot \frac{h}{N^{2}}-\int \tilde{N} \partial_{h}^{2} \mathcal{L}\left(g, \frac{h}{N}\right) \cdot \frac{\dot{h}}{N} \cdot \frac{h}{N}
\end{aligned}
$$

and

$$
\begin{aligned}
\int N \partial_{g} \mathcal{L} & \left(g, \frac{h}{N}\right) \tilde{h} \\
= & \int \partial_{g} \partial_{h} \mathcal{L}\left(g, \frac{h}{N}\right) \cdot h \cdot \tilde{h}-\int \dot{N} \partial_{h}^{2} \mathcal{L}\left(g, \frac{h}{N}\right) \cdot \tilde{h} \cdot \frac{h}{N^{2}} \\
& \quad+\int \partial 2 \frac{2}{h}\left(g, \frac{h}{N}\right) \cdot \tilde{h} \cdot\left(\frac{\dot{h}}{N}\right) .
\end{aligned}
$$

Condition (7.2b) is just the condition for Lagrange's equation for $h / N=k$ which we work out in Theorem 8. 1 below.

For now we want to focus our attention on the nontrivial condition (7.2a). Since we are supposed to have complete degeneracy in $T, \tilde{N}$ is arbitrary, so (7. 2a) is equivalent to

$$
\begin{aligned}
0 \equiv \partial_{g} \mathcal{L}\left(g, \frac{h}{N}\right) \cdot h & -\partial_{g} \partial_{h} \mathcal{L}\left(g, \frac{h}{N}\right) \cdot h \cdot \frac{h}{N} \\
& +\ddot{N} \partial_{h} 2 \mathcal{L}\left(g, \frac{h}{N}\right) \cdot \frac{h}{N} \cdot \frac{h}{N^{2}}-\partial_{h}^{2} \mathcal{L}\left(g, \frac{h}{N}\right) \cdot \frac{h}{N} \cdot \frac{h}{N}
\end{aligned}
$$

Setting $k=h / N$, this becomes

$0 \equiv \partial_{g} \mathcal{L}\left(g, \frac{h}{N}\right) \cdot h-\partial_{g} \partial_{h} \mathcal{L}\left(g, \frac{h}{N}\right) \cdot h \cdot \frac{h}{N}-\partial_{h}^{2} \mathcal{L}\left(g, \frac{h}{N}\right) \cdot \dot{k} \cdot \frac{h}{N}$

Let us take a curve $(\xi(t), g(t), N(t), h(t))$ tangent to $\bar{Z}$ which we suppose exists. Then (7.2c) just says that

$$
\begin{aligned}
& 0 \equiv \frac{\partial}{\partial t}\left[\partial_{h} \mathcal{L}(g, k) \cdot k-\mathcal{L}(g, k)\right] \\
& \text { or } \frac{\partial}{\partial t}\left[\mathcal{H C}(g, k) \mu_{g}\right] \equiv 0 .
\end{aligned}
$$

This proves the theorem.

We shall continue this investigation by showing how to construct $\bar{Z}$ in the next section.

\section{CONSTRUCTION OF THE EQUATIONS FOR A GENERAL LAPSE}

In view of the results of Sec. 7 and the discussion of Sec. 1, we introduce the following "constraint" subset of $T(\mathcal{T} \times \mathfrak{T})$ :

$$
\begin{aligned}
& \mathcal{C} \subset T(T \times \mathscr{T}), \\
& \mathcal{C}=\left\{(\xi, N, g, h): N>0 \text { and } \delta\left[\left(\operatorname{Tr} \frac{h}{N}\right) g-\frac{h}{N}\right]=0\right. \\
& \text { and } \left.\mathcal{H}\left(g, \frac{h}{N}\right)=0\right\} .
\end{aligned}
$$

Since ultimately $N$ will be specified in advance, it is useful to think of $\mathcal{C}$ as a subset of $T \mathfrak{T}$. Unfortunately, at points $g$ of $\mathfrak{C}$ which admit a nontrivial isometry group, $\mathcal{C}$ does not seem to be a manifold. This is 
analogous to the nonmanifold structure of superspace $^{5}$; see also Sec. 11 . However, we shall not require smoothness of $C$ in what follows:

Theorem 8.1: Let $\bar{L}$ on $T(T \times \mathfrak{T})$ be defined as in Sec. 7. Then at points of $e, \bar{L}$ has a Lagrangian vector field $\bar{Z}$. The most general such second order vector field is as follows: Specify an arbitrary curve $\xi(t) \in \mathcal{T}$ and set $N_{t}=d \xi(t) / d t$, assuming $N_{t}>0$. By writing

$$
\bar{Z}(\xi, N, g, h)=(N, \dot{N}, \dot{g}, \dot{h}), \quad k=\frac{h}{N},
$$

then

$$
\left\{\begin{aligned}
\dot{g}=\frac{\partial g_{t}}{\partial t}= & N_{t} k_{t} \\
\dot{k}=\frac{\partial k_{t}}{\partial t}= & N_{t} S_{g_{t}}\left(k_{t}\right)-2 N_{t} \operatorname{Ric}\left(g_{t}\right)+\frac{1}{4} N_{t} \mathfrak{H}\left(g_{t}, k_{t}\right) \\
& +2 \operatorname{Hess} N_{t}
\end{aligned}\right.
$$

Moreover, an integral curve of these equations which begins in $\mathcal{C}$ remains in $\mathcal{C}$.

Proof: With $\bar{Z}$ defined as in the statement of the theorem, we must verify the Lagrangian condition for $\bar{L}$. Referring to the proof of condition 7.1 , this amounts to showing that $(7.2 \mathrm{~b})$ holds and that $(\partial / \partial t)\left[\mathcal{H}(g, k) \mu_{g}\right]=0$ for any integral curve of $\bar{Z}$ starting in $\mathrm{e}$.

From $(7.2 \mathrm{~b})$ it is clear that $\dot{k}$ must be the spray of the DeWitt metric minus the gradient of the potential $V(g)=2 \int N R(g) \mu_{g}$. The equations then follow from Proposition 3.2.

To complete the verification of the Lagrange condition we must show that $(\partial / \partial t)\left[\mathcal{H C}(g, k) \mu_{g}\right]=0$. To do this we first observe that the condition $\delta[(\operatorname{Tr} k) g-k]$ $=0$ is maintained in time; this can be proved exactly as in Sec. 6 by invariance of the Lagrangian under the action of $D$. $D$ must now be considered to act on $\tau$ in the natural way, $D \times T \rightarrow T ; \eta \times \xi \mapsto \xi \circ \eta$. We then prove, under the hypothesis $\delta[(\operatorname{Tr} k) g-k]=0$, that $(\partial / \partial t)\left(\mathcal{K C} \mu_{g}\right)=0$ by a direct verification, analogous to the proof of Proposition 3.4, using the stated equations defined for $\bar{Z}$.

Note: In general, $\bar{Z}$ will not exist at points other than those in $\mathrm{C}$.

The Hessian term is a nonlinear coupling between $N$ and $g$. However, we again assert that the solution for a general $N$ may be obtained from a solution for $N=1$ by integrating a system of ordinary differential equations. This is explained in Sec. 10.

There is another interesting way to see that one has $(\partial / \partial t)\left(\mathcal{H} \mu_{g}\right)=0$ for any theory invariant under the full relativistic time translation group $\tau$. This is an alternative approach to that used in 7.1 although it is not detailed enough to allow for the construction of the equations of motion. It does, however, provide a group theoretical argument for the relationship between $(\partial / \partial t)\left(\mathcal{K} \mu_{g}\right)=0$ and time translation invariance (in the relativistic sense).

Proposition 8.1 : Let $\mathscr{L}$ be any Lagrangian density on $\mathfrak{M}$ (or any function-space for that matter) with extension to $\overline{\mathcal{L}}$ on $T(T \times \mathscr{T})$ as defined in Sec. 7 . Suppose

$$
\bar{L}=\int N \mathcal{L}\left(g, \frac{h}{N}\right) \mu_{g}
$$

has a Lagrangian vector field $\bar{Z}$ on some subspace $\mathfrak{C} \subset T(T \times \mathscr{T})$. Let $\mathfrak{C}$ be invariant under relativistic time translations (see below), and let integral curves of $\bar{Z}$ map e to e. Then along such integral curves, $(\partial / \partial t)\left(\mathcal{H} \mu_{g}\right) \equiv 0$.

Proof: $T$ is a vector space and as an additive group, acts on $T \times \mathfrak{T}$ and $\mathrm{e}$ in a natural way. For $\xi_{0} \in \tau^{\prime}$ we get a map of $\mathcal{T} \times \mathfrak{T} \rightarrow \tau \times \mathfrak{T}$ by $(\xi, g) \mapsto$ $\left(\xi+\xi_{0}, g\right)$. There is a corresponding one parameter group $\Phi_{t}(\xi, g)=\left(\xi+t \xi_{0}, g\right)$. This is generated by the vector field $(\xi, g) \mapsto\left(\xi_{0}, 0\right)$.

Now the tangent action of $\Phi_{t}$ leaves $\bar{L}$ invariant, since $T \Phi_{t}(\xi, N, g, h)=\left(\xi+t \xi_{0}, N, g, h\right)$ and $\bar{L}$ depends only on $N$, not on $\xi$. Thus we may apply Proposition 2.3 . By a straightforward computation, we find that the fiber derivative is given as follows:

$$
\begin{aligned}
& F \bar{L}(\xi, N, g, h): T_{(\xi, g)}(\mathcal{T} \times \mathfrak{T}) \rightarrow \mathbf{R}, \\
& (\bar{N}, \bar{h}) \mapsto-\int_{M} \bar{N} \mathscr{C}\left(g, \frac{h}{N}\right) \mu_{g}+\int_{M}\left[\partial_{h} \mathcal{L}\left(g, \frac{h}{N}\right) \cdot \bar{h}\right] \mu_{g} .
\end{aligned}
$$

Thus with $(\bar{N}, \bar{h})=\left(\xi_{0}, 0\right)$ we conclude from proposition 2.3 that

$$
\int_{M} \xi_{0} \mathfrak{H C} \mu_{g}
$$

is a constant of the motion. Since $\xi_{0}$ is arbitrary, the result follows.

Observe that $\mathcal{F}^{\mathrm{C}}$ is not the energy density for $\bar{L}$ but rather is that for $L$. Since $\bar{L}$ is homogeneous, its associated energy function is identically zero; since $\omega_{\bar{L}}$ is degenerate, this does not imply trivial equations of motion. Finally note that the requirement $(\delta \pi)^{b}=0$ is buried in Proposition 8.1 through the assumptions that $\bar{Z}$ exists and integral curves stay in $C$. Thus Proposition 8.1 is just illustrative, with the main results in Theorems 7.1 and 8.1 .

We prefer the proofs we have given for the maintenance of the supplementary conditions since they are natural consequences of the Hamiltonian structure of the evolution equations and their dynamical symmetries. Moreover, in this approach we need not rely on identities in the corresponding four geometry.

\section{RELATIONSHIP WITH THE FOUR GEOMETRY}

In this section we establish the equivalence between the Einstein system (E), with a given lapse $N_{t}$ and shift $X_{t}$, for the evolving three geometry $g_{i j}$ and the Ricci flatness of the Lorentz metric $g^{L}$ constructed on $I \times M[I=(-\epsilon, \epsilon)]$; the metric $g^{L}$ is obtained by decreeing that $(1 / N, X / N)$ be a unit timelike vector field on $I \times M$ orthogonal to the $\{t\} \times M$ hypersurfaces. To satisfy this condition, we construct $g^{L}$ from $g_{t}, X_{t}$, and $N_{t}$ as follows:

$$
\begin{aligned}
g L(t, m) & \left(\left(r, v_{m}\right),\left(s, w_{m}\right)\right) \\
& =g_{t}(m) \cdot\left(v_{m}-r X_{t}(m), w_{m}-s X_{t}(m)\right)-r s N_{t}^{2}(M) .
\end{aligned}
$$

In coordinates, this formula reads

${ }^{4} g_{\alpha \beta} d x^{\alpha} d x^{\beta}=\left(X^{i} X_{i}-N^{2}\right)(d t)^{2}-2 X_{i} d x^{i} d t+g_{i j} d x^{i} d x^{j}$, 
where $x^{\alpha}=\left(t, x^{i}\right)$ and $X_{i}=g_{i j} X^{j}$. We are assuming that $X_{t}$ has length less than $N_{t}$ which means that our observer has velocity less than that of light, relative to a Gaussian reference system.

Theorem 9.1: Let $X_{t}$ and $N_{t}$ be a given lapse and shift. Then a curve $g_{t} \in \mathfrak{T}$ satisfies the system (E) with lapse and shift $N_{t}$ and $X_{t}$ if and only if the Lorentz metric $g^{L}$ constructed above is Ricci flat; i.e., $R_{\alpha \beta}=0$.

Obviously this theorem is basic to the whole program and is in Ref. 1 for $N=1$ and $X=0$. Here we are interested in the situation for arbitrary $N$. One interesting feature is to see how the Hessian term of $N$ in the equations of evolution arises. We have seen in Secs. 3 and 8 how it arose in the Lagrangian formulation.

The proof of Theorem 9.1 is based on a decomposition of the Riemann-Christoffel curvature tensor in terms of quantities associated with an embedded hypersurface. Four of the equations are the GaussCodazzi equations which relate the curvature tensor of $I \times M$ to the curvature tensor and second fundamental form $S$ of the embedded hypersurface $M$. The other six equations involve more than the geometry of $M$ and $S$; they depend also on a family of embeddings. A convenient reference for this result is Yano ${ }^{21}$ Chap. ${ }^{5}$; see also, Abraham, 22 Sec. 9. For the purposes of this paper we shall translate the formulas into coordinate notation. In doing this we choose a coordinate system in which the $t$-axis is normal to the hypersurface $M$; in other words, we assume that the unit timelike normal is of the form $Z=\left(Z^{0}, 0\right)$ so that $g_{0 k}=0$.

Thus $g_{\alpha \beta}$ is of the form $-N^{2} d t^{2}+g_{i j} d x^{i} d x^{j}$ and $Z=(1 / N, 0)$. The case of an arbitrary shift $X_{t}$ may be dealt with by the methods explained in Secs. 4 and 5.

Lemma 9.1: Let $M_{t}$ be a family of three manifolds embedded as spacelike hypersurfaces in a Lorentz manifold $V$. Let ${ }^{4} R_{a \beta \gamma \delta}$ be the curvature tensor on $V$ and ${ }^{3} R_{i j k l}$ that on $M$. Let $S_{i j}$ be the second fundamental form ("extrinsic curvature") of $M$ and $Z$ the unit normal to $M$. Then in a coordinate system in which $Z=(1 / N, 0)$, we have the following decomposition of ${ }^{4} R_{\alpha \beta \gamma \delta}$ :

(iii) $4 R_{i j k 0}=S_{k i \mid j}-S_{k j \mid i}$,

where all covariant derivatives are taken in the metric on $M$.

Proof: The decomposition (ii) is the Gauss equation (Yano, ${ }^{21}$ p. 94)

$$
\begin{aligned}
{ }^{4} R(X, Y, U, W)={ }^{3} R(X, Y, U, W)+ & {[S(X, W) S(Y, U)} \\
& -S(Y, W) S(X, U)]
\end{aligned}
$$

written in coordinates. There is a change of sign over what is in Yano because $g(Z, Z)=-1$ rather than +1 . The decomposition (ii) holds generally for any hypersurface and is a direct consequence of the rela- tionship between the induced connection ${ }^{3} \nabla$ and the second fundamental form $S$,

$$
{ }^{4} \nabla_{X} Y={ }^{3} \nabla_{X} Y+S(X, Y) Z
$$

and the definition of the Riemann-Christoffel curvature tensor. Similarly (iii) is the Codazzi equation (Yano, ${ }^{21}$ p. 95)

$$
R(X, Y, U, Z)=\nabla_{Y} S(X, U)-\nabla_{X} S(Y, U) .
$$

The decomposition (i) involves the geometry of $M, S$, and the family of embeddings (otherwise $\partial S_{i j} / \partial t$ has no meaning). The decomposition (i) may be deduced (by a long computation) from Abraham ${ }^{22}$ Sec. 9, but we can also give a direct proof as follows. Now we can write

$$
g_{\alpha \beta} d x^{\alpha} d x^{\beta}=-N^{2} d t^{2}+g_{i j} d x^{i} d x^{j}
$$

and compute directly from $\Gamma_{\mu \nu}^{\alpha}=\frac{1}{2} g^{\lambda \alpha}\left(g_{\mu \lambda, \nu}+g_{\nu \lambda, \mu}\right.$ $\left.-g_{\mu \nu, \lambda}\right)$ that

$$
\begin{array}{ll}
\Gamma_{00}^{0}=N_{, 0} / N, & \Gamma_{i 0}^{0}=N_{, i} / N, \\
\Gamma_{i j}^{0}=g_{i j, 0} / 2 N^{2}, & \Gamma_{0 j}^{k}=\frac{1}{2} g^{l k} g_{l j, 0}, \\
\Gamma_{00}^{i}=N g^{j i} N_{, j}, & { }^{4} \Gamma_{j k}^{i}={ }^{3} \Gamma_{j k}^{i} .
\end{array}
$$

Contracting (9.1) with $Z$ gives

$$
S(X, Y)=-\left({ }^{4} \nabla_{X} Y, Z\right)=\left({ }^{4} \nabla_{X} Z, Y\right) .
$$

Thus in coordinates,

$$
S_{i j}=Z_{i \mid j}=Z_{i, j}-\Gamma_{i j}^{\alpha} Z_{\alpha}=\Gamma_{i j}^{0} N=g_{i j, 0} / 2 N,
$$

since $Z_{\alpha}=(-N, 0)$.

Now by definition

$R(Z, X, Z, Y)=\left\langle\nabla_{Z} \nabla_{X} Z, Y\right\rangle-\left\langle\nabla_{X} \nabla_{Z} Z, Y\right\rangle-\left\langle\nabla_{[X, Z]} Z, Y\right\rangle$, so that

$$
\frac{1}{N^{2}} R_{0 i 0 j}=R(Z, X, Z, Y),
$$

where $Z=((1 / N)(\partial / \partial t), 0), X=\left(0, \partial / \partial x^{i}\right)$, $Y=\left(0, \partial / \partial x^{j}\right)$. Now one easily computes the following:

$$
\begin{aligned}
& \left(\nabla_{X} Z\right)^{0}=S_{i}^{j}, \\
& \left(\nabla_{X} Z\right)^{0}=-\frac{1}{N^{2}} N_{, i}+\Gamma_{i 0}^{0} \frac{1}{N}=0, \\
& \left(\nabla_{Z} W\right)^{k}=W_{, 0}^{k} / N+S_{j}^{k} W^{j}+g^{j k} N_{, j} W^{0} .
\end{aligned}
$$

So we get

$$
\begin{aligned}
\left\langle\nabla_{Z} \nabla_{X} Z, Y\right\rangle & =g_{j l}\left(\nabla_{Z} \nabla_{X} Z\right)^{l} \\
& =g_{j l} S_{i, 0}^{l} / N+S_{k}^{l} S_{i}^{k} g_{j l} \\
& =\frac{1}{N} \frac{\partial}{\partial t}\left(S_{i j}\right)-(S \times S)_{i j} .
\end{aligned}
$$

Similarly,

$\left\langle\nabla_{X} \nabla_{Z} Z, Y\right\rangle=\left(N_{, j} / N\right)_{\mid i}=N_{|j| i} / N-N_{, j} N_{, i} / N^{2}$

and finally since $[X, Z]^{k}=0,[X, Z]^{0}=N_{, i} / N^{2}$, we get

$$
\left\langle\nabla_{[X, Z]} Z, Y\right\rangle=N_{, i} N_{, j} / N^{2}
$$

Thus adding up (9.2), (9.3) and (9.4) yields (i). 
In terms of $k_{i j}=(1 / N)\left(\partial g_{i j} / \partial t\right)=2 S_{i j}$, we have

$$
\left\{\begin{aligned}
{ }^{4} R_{0 i 0 j} & =N^{2}\left(\frac{1}{2 N} \frac{\partial k_{i j}}{\partial t}-\frac{1}{4}(k \times k)_{i j}-\frac{N_{|i| j}}{N}\right), \\
{ }^{4} R_{i j k l} & ={ }^{3} R_{i j k l}+\frac{1}{4}\left(k_{i l} k_{j k}-k_{i k} k_{j l}\right), \\
{ }^{4} R_{i j k 0} & =\frac{1}{2}\left(k_{k i \mid j}-k_{k j \mid i}\right) .
\end{aligned}\right.
$$

Proof of Theorem 9.1: We prove Theorem 9.1 for the case of $X_{t}=0$. The general case is handled using the methods of Secs. 4 and 5 . So suppose that $g_{\alpha \beta} d x^{\alpha} d x^{\beta}$ $=-N^{2} d t^{2}+g_{i j} d x^{i} d x^{j}$ is Ricci flat. Using Yano's conventions, the Ricci tensor is

$$
{ }^{4} R_{\alpha \beta}={ }^{4} g{ }^{\gamma \delta}{ }^{4} R_{\gamma \alpha \beta \delta}=-{ }^{4} g{ }^{\gamma \delta} R_{\gamma \alpha \delta \beta^{*}} .
$$

We have

$$
\begin{aligned}
0= & { }^{4} R_{i j}=-{ }^{4} g^{\alpha \beta}{ }^{4} R_{\alpha i \beta j}=\frac{1}{N^{2}}{ }^{4} R_{0 i 0 j}-g^{k l}{ }^{4} R_{k i l j} \\
= & \left(\frac{1}{2 N} \frac{\partial k}{\partial t}-\frac{1}{4} k \times k-\frac{1}{N}(\operatorname{Hess} N)\right)+\{\operatorname{Ric}(g) \\
& \left.-\frac{1}{4}[k \times k-(\operatorname{Tr} k) k]\right\}
\end{aligned}
$$

where we have used decomposition (ii) as well as (i). This gives the required equation for $\partial k / \partial t$.

Similarly using decomposition (iii) we have $0={ }^{4} R_{0 i}$ $=-4_{g} \alpha \beta{ }^{4} R_{\alpha i \beta O}=-{ }^{3}{ }^{k l}{ }^{4} R_{k i \ell 0}=\frac{1}{2}\left(k_{i j}^{j}-k j_{j i}\right)$ $=-\frac{1}{2} \delta(k-(\operatorname{Tr} k) g)$, which gives the divergence condition.

Finally using decomposition (i) again, we have

$$
\begin{aligned}
0 & ={ }^{4} R_{00}=-{ }^{4} g^{\alpha \beta}{ }^{4} R_{\alpha 0 \beta 0}=-3 g^{k l}{ }^{4} R_{0 k 0 l} \\
& =-{ }^{3} g^{k l} N^{2}\left(\frac{1}{2 N} \frac{\partial k_{k l}}{\partial t}-\frac{1}{4}(k \times k)_{k l}-\frac{(\operatorname{Hess} N)_{k l}}{N}\right) .
\end{aligned}
$$

If we now substitute the equation for $\partial k / \partial t$ in this expression, it simplifies down to $\frac{1}{2} N^{2} \mathfrak{H}(g, k)$ $=\frac{1}{2} N^{2}[\mathcal{K}+2 R(g)]$ so we get the energy condition.

The converse of the theorem is proved by retracing the steps.

\section{THE INTRINSIC SHIFT VECTOR FIELD}

In this section we study the relationship between solutions of the Einstein system (E) with the same Cauchy data but with different prescribed lapse functions. We suppose that we have a solution $\left(g_{t}, k_{t}\right)$, $|t|<\epsilon$ of (E) for a given lapse $N_{t}$ and shift vector field $X_{t}=0$, and we wish to find the solution $\left(\bar{g}_{t}, \bar{k}_{t}\right)$ to $(E)$ with lapse $\bar{N}=1$ and $\bar{X}=0$ such that $\left(\bar{g}_{0}, \bar{k}_{0}\right)$ $=\left(g_{0}, k_{0}\right)$. The converse problem of finding $\left(g_{t}, k_{t}\right)$ for an arbitrary lapse $N$ given the solution $\left(\bar{g}_{t}, \bar{k}_{t}\right)$ for $\bar{N}=1$ proceeds similarly (see Theorem 10. 3 below).

The above problem is well known to be equivalent to finding the Gaussian normal coordinates for the Lorentz metric $g_{\alpha \beta} d x^{\alpha} d x^{\beta}=-N^{2} d t^{2}+g_{i j} d x^{i} d x j$. What we wish to do is geometrize this situation a bit.

Lemma 10.1: Let $N_{t}$ be an arbitrary lapse function, let $g_{t} \in \mathfrak{M},|t|<\epsilon$, be a one-parameter curve of metrics, and let $g^{L}(t, m) \cdot\left[\left(r, v_{m}\right)\left(s, w_{m}\right)\right]=-N_{t}^{2}(m) r s$ $+g_{t}(m) \cdot\left(v_{m}, w_{m}\right)$ be the associated Lorentz metric on $I \times M, I=(-\epsilon, \epsilon)$. Then there exists a unique curve $\tau_{t} \in \mathcal{T}^{\prime},|t|<\epsilon^{\prime} \leq \epsilon$ with $\tau_{0}=0$ such that

$$
\frac{d \tau}{d t}=N_{t} \sqrt{1+\left\|\operatorname{grad} \tau_{t}\right\|},
$$

where $\left(\operatorname{grad} \tau_{t}\right) j=g^{i j}\left(d \tau / d x^{i}\right)$ is computed with respect to the time-dependent metric $g_{t}$. The function $\tau: I \times M \rightarrow \mathbf{R}, \tau(t, m) \mapsto \tau_{t}(m)$ is the proper time from $(t, m)$ to $\{0\} \times M$ measured backwards along a unit timelike geodesic normal to $\{0\} \times M$.

Proof: Equation (10.1) is just the eikonal equation $-\frac{1}{N_{t}^{2}}\left(\frac{\partial \tau}{\partial t}\right)^{2}+\|\operatorname{grad} \tau\|^{2}=-1=g^{\alpha \beta} \frac{\partial \tau}{\partial x^{\alpha}} \frac{\partial \tau}{\partial x_{\beta}}$,

which is a single first order nonlincar partial differential equation. By the Cauchy method of characteristics, this single equation can be reduced to a system of eight ordinary differential equations in Hamiltonian form, namely the geodesic equations of $g_{\mu \nu}$ :

$$
\left\{\begin{array}{l}
\frac{d x^{u}}{d t}=g^{\mu \nu} p_{\nu}=\frac{\partial H}{\partial p_{\mu}}, \\
\frac{d p_{\mu}}{d \tau}=-\frac{1}{2} \frac{\partial g^{\alpha \beta}}{\partial x^{\mu}} p_{\alpha} p_{B}=-\frac{\partial H}{\partial x^{\mu}},
\end{array}\right.
$$

where the Hamiltonian $H=\frac{1}{2} g{ }^{\mu} p_{\mu} p_{\nu}$.

From the initial condition $\tau(0, m)=0$ and $\mathrm{Eq}$. (10.2) itself, we conclude that $(d \tau / d t)(0, m)=N_{0}(m)$. System (10.3) can be integrated for short time $0<\epsilon^{\prime} \leq \epsilon$ subject to the initial conditions $x^{\mu}(0, m)=(0, m)$ and $p_{\mu}(0, m)=\left(N_{0}(m), 0\right)$ to give $\left(x^{\mu}(\tau, m), p_{\mu}(\tau, m)\right)$. Since the hypersurface $\{0\} \times M$ is noncharacteristic, $x^{\mu}(\tau, m)$ can be inverted for $|t|<\epsilon^{\prime \prime} \leq \epsilon^{\prime} \leq \epsilon$ to give a function $\tau\left(x^{\mu}\right)$ which satisfies $\tau(0, m)=0$, Eq. (10.2), and $\partial \tau / \partial x^{\mu}=p_{\mu}$. That the geodesics are unit timelike geodesics follows from conservation of $2 \mathrm{H}$ $=g^{\alpha \beta} p_{\alpha} p_{\beta}=-1$ and since $d x^{\mu} / d t(0, m)=g^{\mu \nu} p_{\nu}(0, m)$ $=g^{\mu \nu}\left(N_{0}(m), 0\right)=\left(-\left(1 / N_{0}(m), 0\right)\right.$, they are normal to $\{0\} \times M$.

The factor $\sqrt{1+\|\operatorname{grad} \tau\|^{2}}$ in the expression for $d \tau / d t$ takes into account the fact that, in general, the lapse depends on space coordinates and therefore "pushes" up the hypersurface $\{0\} \times M$ unevenly along $\tau=$ constant hypersurfaces in $I \times M$.

There is another way of looking at the lapse function which has been given by Wheeler ${ }^{1}$ : Namely it is trivial to check that

$$
\xi_{t}(m)=\int_{0}^{t} N_{\lambda}(m) d \lambda
$$

is the proper time from $\{0\} \times M$ to $\{l\} \times M$ measured along the curve $\lambda \mapsto(\lambda, m)$ for $m$ fixed. This differs from $\tau_{t}(m)$ in that $\tau_{t}(m)$ is the proper time for an observer following a geodesic; that is, one in free fall. The curve $\lambda \mapsto(\lambda, m)$ is not a geodesic because of the spatial dependence of $N$.

For the rest of this section, we will refer to $\tau_{t}$ as computed from Lemma 10.1 as the proper time function associated with $N_{t}$ and $g_{t}$. Note that $\tau_{t}$ is just the time part $\bar{t}\left(t, x^{\prime}\right)$ of the coordinate transformation $\bar{x}^{\alpha}\left(x^{\mu}\right)$ which transforms $g_{\alpha \beta} d x^{\alpha} d x^{\beta}=-N^{2} d t^{2}$ $+g_{i j} d x^{i} d x^{j}$ to Gaussian coordinates, as can be seen from the eikonal equation for $g_{\alpha \beta}$,

$$
-1=-\frac{1}{N^{2}} \frac{\partial \bar{t}}{\partial t}^{2}+g^{k l} \frac{\partial \bar{t}}{\partial x^{k}} \frac{\partial \bar{t}}{\partial x^{l}} .
$$

The question naturally arises if we can construct the rest of the Gaussian coordinate system from $\tau_{t}$ alone. Let $\phi_{t}(m)=\bar{x}^{i}\left(l, x^{j}\right)$ denote the spatial part of the 
transformation to Gaussian coordinates, so that $\left(\tau_{t}(m), \phi_{t}(m)\right)=\bar{x}^{\mu}\left(x^{\alpha}\right)$ is the coordinate transformation leading to Gaussian coordinates. Since $\phi_{t}$ is a diffeomorphism, it is the flow of a time-dependent vector field on $M$, so that it behaves just like a shift $\eta_{t}$ generated by a shift vector field $X_{t}$. We call $\phi_{t}$ the intrinsic shift and its generator $Y$ as the intrinsic shift vector field of $N$. Theorem 10.1 below gives a way to compute $\phi_{t}$ or $Y_{t}$ from the proper time function $\tau_{t}$ alone.

In the transformation to Gaussian coordinates, the hypersurface $\{t\} \times M$ is mapped into the hypersurface $\tau^{-1}(t)$, so $\phi_{t}(m)$ is the spatial coordinate of $(t, m)$ in the Gaussian coordinate system. The intrinsic shift thus describes the shifting of the spatial coordinates in the $\{t\} \times M$ hypersurface due to the fact that the lapse $N_{t}$ depends on the space variable so that each point of $\{t\} \times M$ does not have the same proper time coordinate $\tau_{t}(m)$. Thus the hypersurface $\{t\} \times M$ is tilted when it is stretched to fit the $\tau=$ constant contours. This tilting causes a shifting of the spatial coordinates in $\{t\} \times M$ which is described by the intrinsic shift $\phi_{t}$.

Theorem 10.1: Let $N_{t}$ and $g_{t}$ be given and let $\tau_{t}$ be determined from Lemma 10.1 . Let $Z_{t}$ be the timedependent vector field defined by

$$
Z_{t}=-\frac{N_{t}}{\sqrt{1+\left\|\operatorname{grad} \tau_{t}\right\|^{2}}} \operatorname{grad} \tau_{t}
$$

and let $\psi_{t}, \psi_{0}=i d_{M}$ be its flow. Then the intrinsic shift $\phi_{t}$ is given by $\phi_{t}=\psi_{t}^{-1}$ and the intrinsic shift vector field is

$$
Y_{t}=-\left(\psi_{t}^{-1}\right)_{*} Z_{t}=-\left(\phi_{t}\right)_{*} Z_{t}
$$

Proof: Let $\phi_{t}$ be the space part of the transformation to the Gaussian coordinate system. Then the condition on $\phi_{t}$ comes from requiring that the $g^{0 i}$ components of $g^{L}$ remain zero in the Gaussian coordinate system. This condition is

$$
0=-\frac{1}{N^{2}} \frac{\partial \tau}{\partial t} \frac{\partial \bar{x}^{i}}{\partial t}+g^{k l} \frac{\partial \tau}{\partial x^{k}} \frac{\partial \bar{x}^{i}}{\partial x^{l}}
$$

This condition is rewritten as

$$
\begin{aligned}
\frac{\partial \tau_{t}}{\partial t} \frac{\partial \phi_{t}^{i}}{\partial t} & =N_{t}^{2} g_{t}^{k l} \frac{\partial \phi_{t}^{i}}{\partial x^{l}} \frac{\partial \tau_{t}}{\partial x^{k}}=N_{t}^{2} T \phi_{t} \cdot \operatorname{grad} \tau_{t} \\
& =\left(\frac{d \tau_{t}}{d t}\right)^{2} \frac{1}{1+\left\|\operatorname{grad} \tau_{t}\right\| 2} T \phi_{t} \cdot \operatorname{grad} \tau_{t}
\end{aligned}
$$

so that

$$
\begin{aligned}
\frac{d \phi_{t}}{d t} & =\left(\frac{d \tau_{t}}{d t}\right)\left(\frac{1}{1+\left\|\operatorname{grad} \tau_{t}\right\| 2}\right) T \phi_{t} \cdot \operatorname{grad} \tau_{t} \\
& =\frac{N_{t}}{\sqrt{1+\left\|\operatorname{grad} \tau_{t}\right\|^{2}}} T \phi_{t} \cdot \operatorname{grad} \tau_{t} \\
& =-T \phi_{t} \circ Z_{t^{*}}
\end{aligned}
$$

Thus $\phi_{t}$ satisfies

$$
\frac{d \phi_{t}}{d t} \circ \phi_{t}^{-1}=-T \phi_{t^{\circ}} Z_{t^{\circ}} \phi_{t}^{-1}=-\left(\phi_{t}\right)_{*} Z_{t}
$$

so that $-\left(\phi_{t}\right)_{*} Z_{t}$ is the generator of $\phi_{t}$. Let $\psi_{t}, \psi_{0}$ $=i d_{M}$ be the flow of $Z_{t}$. Then from Lemma 4.2 , - $\left(\psi_{t}^{-1}\right)_{*} Z_{t}$ has the flow $\psi_{t}^{-1}$ so that $\phi_{t}=\psi_{t}^{-1}$. Thus $-\left(\phi_{t}\right)_{*} Z_{t}=-\left(\psi_{t}^{-1}\right)_{*} Z_{t}=Y_{t}$ is the intrinsic shift vector field associated with $N$.

We now consider how solutions $\left(g_{t}, k_{t}\right),|t|<\epsilon$ of the Einstein system (E) with an arbitrary lapse $N_{t}$ are related to solutions $\left(\bar{g}_{t}, \bar{h}_{t}\right),|t|<\epsilon^{\prime} \leq \epsilon$ of $(\mathrm{E})$ with the same Cauchy data and $\bar{N}=1$. As before, this is equivalent to finding how the space part of $g_{\alpha \beta} d x^{\alpha} d x^{\beta}$ $=-N^{2} d t^{2}+g_{i j} d x^{i} d x^{j}$ transforms when we transform to Gaussian coordinates. This is also equivalent to finding the metric $\tilde{g}_{\tau}$ induced on the $\tau=$ constant hypersurfaces in the space $I \times M$ with Lorentz metric $g_{\alpha B}$.

The fact that $g_{t}$ is a solution of $(E)$ is again peripheral as we are just computing how the space part of a Lorentz metric of the form $g_{\alpha \beta} d x^{\alpha} d x^{\beta}=-N^{2} d t^{2}$ $+g_{i j} d x^{i} d x^{j}$ transforms when we transform to Gaussian coordinates.

Theorem 10.2: Let $N_{t}$ and $g_{t}$ be given, $|t|<\epsilon$ and let $\tau_{t}$ be determined from Lemma 10,1. Let $\psi_{t}, \psi_{0}=i d_{M}$ be the flow of $Z_{t}=-N_{t}\left(1+\left\|\operatorname{grad} \tau_{t}\right\|^{2}\right)^{-1 / 2}$ $\operatorname{grad} \tau$; let $\phi_{t}=\psi_{t}^{-1}$ be the intrinsic shift; and let $Y_{t}=-\left(\phi_{t}\right)_{*} Z_{t}$ be the instrinsic shift vector field associated with $N_{t}$.

Let $g_{l}^{-1}=g^{i j}$ be $g_{t}$ in contravariant form and let

$$
\begin{aligned}
& \bar{g}^{-1}\left(\tau_{t}(m), \phi_{t}(m)\right) \\
& =T \phi_{t} \otimes T \phi_{t}\left(g_{t}^{-1}-\frac{Z_{t}}{N_{t}} \otimes \frac{Z_{t}}{N_{t}}\right)(m)=T \phi_{t} \otimes T \phi_{t} \\
& \quad\left(g_{t}^{-1}-\frac{\operatorname{grad} \tau_{t}}{\sqrt{1+\left\|\operatorname{grad} \tau_{t}\right\|^{2}}} \otimes \frac{\operatorname{grad} \tau_{t}}{\sqrt{1+\left\|\operatorname{grad} \tau_{t}\right\|^{2}}}\right)(m) \\
& =T \phi_{t} \otimes T \phi_{t}\left(g_{t}^{-1}\right)(m)-\frac{Y_{t}}{N_{t}} \otimes \frac{Y_{t}}{N_{t}}\left(\phi_{t}(m)\right) .
\end{aligned}
$$

Then $\overline{g_{\tau}}$ is the metric induced on $\tau=$ constant hypersurfaces by the Lorentz metric $-N^{2} d t^{2}+g_{i j} d x^{i} d x^{j}$. As above, denoting the Gaussian coordinates by $\left(\tau\left(t, x^{i}\right), \phi^{i}(t, x)\right)$ we have the coordinate expression

$$
\begin{aligned}
& \bar{g}^{i j}=\frac{\partial \phi^{i}}{\partial x^{k}} \frac{\partial \phi^{j}}{\partial x^{l}} \\
& \times\left(g^{k l}-\left(1+g^{a b} \frac{\partial \tau}{\partial x^{a}} \frac{\partial \tau}{\partial x^{b}}\right)^{-1} g^{k m_{g} \ln } \frac{\partial \tau}{\partial x^{m}} \frac{\partial \tau}{\partial x^{n}}\right)(t, x) .
\end{aligned}
$$

Remark: Note that in order that $\bar{g}^{-1}$ remain positive definite,

$$
g_{t}(X, X)>\left(\frac{d \tau \cdot X}{\sqrt{1+\left\|\operatorname{grad} \tau_{t}\right\| 2}}\right)^{2} .
$$

This holds at $t=0$ and so will hold for some $t$-interval around 0 .

Proof: Let $-N^{2} d t^{2}+g_{i j} d x^{i} d x^{j}$ be the Lorentz metric associated with $g_{t}$ and $N_{t}$. Transforming this metric to Gaussian coordinates gives the transformation law for the 3-metric $g^{i j}$ as

$$
\begin{aligned}
\bar{g}^{i j}\left(\gamma\left(t, x^{k}\right), \bar{x}^{i}\left(t, x^{k}\right)\right)=\frac{\partial \bar{x}^{i}}{\partial x^{m}}\left(t, x^{k}\right) & \frac{\partial \bar{x}^{j}}{\partial x^{n}}\left(t, x^{k}\right) g^{m n}\left(t, x^{k}\right) \\
& -\frac{1}{N^{2}} \frac{\partial \bar{x}^{i}}{\partial t}\left(t, x^{k}\right) \frac{\partial \bar{x}^{j}}{\partial t}\left(t, x^{k}\right) .
\end{aligned}
$$


By using

$$
\frac{1}{N} \frac{\partial \bar{x}^{i}}{\partial t}=\frac{N}{\partial \tau / d t} \frac{\partial \bar{x}^{i}}{\partial x^{l}} g^{k l} \frac{\partial \tau}{\partial x^{k}}
$$

from the proof of Theorem 10.1 ,

we have

$\bar{g}^{i j}\left(\tau\left(t, x^{k}\right), \bar{x}^{i}\left(t, x^{k}\right)\right)$

$=\frac{\partial \vec{x}^{i}}{\partial x^{m}} \frac{\partial \bar{x} j}{\partial x^{m}}\left(g^{m n}-\frac{1}{1+\|\operatorname{grad} \tau\|^{2}} g^{m k} \frac{\partial \tau}{\partial x^{k}} g^{n l} \frac{\partial \tau}{\partial x^{l}}\right)\left(t, x^{k}\right)$

which is written as

$$
\begin{aligned}
& \bar{g}^{-1}\left(\tau_{t}(m), \phi_{t}(m)\right)=T \phi_{t} \otimes T \phi_{t} \\
& \quad \times\left(g_{t}^{-1}(m)-\frac{\operatorname{grad} \tau_{t}(m)}{\sqrt{1+\left\|\operatorname{grad} \tau_{t}\right\|^{2}}} \otimes \frac{\operatorname{grad} \tau_{t}(m)}{\sqrt{1+\left\|\operatorname{grad} \tau_{t}(m)\right\|^{2}}}\right) .
\end{aligned}
$$

As a corollary we get an expression for the solution to the Einstein system (E) for $\bar{N}=1$, given a solution $\left(g_{t}, k_{t}\right)$ for arbitrary lapse $N_{t}$.

Corollary 10.1: Let $\left(g_{t}, k_{t}\right)$ be a solution to the Einstein system $(\mathrm{E})$ with $N_{t}$ given and $X_{t}=0$. Let $\tau_{t}, \tau_{0}=0$ be determined from Lemma 10.1 , let $Z_{t}=-N_{t} /\left(1+\| \operatorname{grad} \tau_{t} \mid 2\right)^{-1 / 2} \operatorname{grad} \tau_{t}$ and let $\phi_{t}$ be the intrinsic shift. Let $g_{t}^{-1}$ be $g_{t}$ in contravariant indices; let

$$
\begin{aligned}
& Y_{t}=-\left(\phi_{t}\right)_{*} Z_{t}, \\
& \bar{g}^{-1}\left(\tau_{t}(m), \phi_{t}(m)\right)=T \phi_{t} \otimes T \phi_{t}\left(g_{t}^{-1}-\frac{Y_{t}}{N_{t}} \otimes \frac{Y_{t}}{N_{t}}\right)(m),
\end{aligned}
$$

and let $\bar{k}_{\tau}=\partial \bar{g}_{\tau} / \partial \tau$. Then $\left(\bar{g}_{\tau}, \bar{k}_{\tau}\right)$ is a solution to $(\mathrm{E})$ with $\bar{N}_{t}=1$ and

$$
\left(\bar{g}_{0}, \bar{k}_{0}\right)=\left(\bar{g}_{0}, k_{0}\right) \text {. }
$$

Proof: From the theorem, $\bar{g}_{\tau}$ is the metric induced on $\tau=$ constant hypersurfaces and so is the solution of $(E)$ in Gaussian coordinates; that is, with $\bar{N}_{t}=1$.

Since $\tau_{0}=0, \bar{g}_{0}=g_{0}$. From the chain rule,

$$
\left.\frac{\partial \bar{g}^{-1}}{\partial \tau} \frac{\partial \tau}{\partial t}\right|_{t=0}=\left.\frac{\partial \bar{g}^{-1}}{\partial t}\right|_{t=0} \text {. }
$$

Since $Y_{0}=0$ and $\phi_{0}=i d_{M}$, one sees from the above expression for $\bar{g}^{-1}$ that

$$
\left.\frac{\partial \bar{g}^{-1}}{\partial t}\right|_{t=0}=\left.\frac{\partial g^{-1}}{\partial t}\right|_{t=0} \text {. }
$$

Thus

$$
\left.\frac{\partial \bar{g}^{-1}}{\partial \tau} \frac{\partial \tau}{\partial t}\right|_{t=0}=\left.\frac{\partial g^{-1}}{\partial t}\right|_{t=0},
$$

or $N_{0} \bar{k}_{0}=N_{0} k_{0}$, so that $\bar{k}_{0}=k_{0}$.

In case the lapse $N_{t}=N(t)$ does not depend on the space coordinate, then $N_{t}=d \tau_{t} / d t$ and the relation of solutions $\left(g_{t}, k_{t}\right)$ to Einstein's equations with $N(t)$, $X=0$, and $\bar{N}_{t}=1, X=0$ is particularly simple. In

fact, if we define $\tau(t)=\int_{0}^{t} N(\lambda) d \lambda$, then the solutions to the Einstein equations with $\bar{N}=1, X=0$ are just reparameterizations by $\tau(t)$ of the solutions $\left(g_{t}, k_{t}\right)$ of the Einstein system with $N_{t}=N(t), X=0$. We check this formally as follows:
Proposition 10.1: Let $N_{t}=N(t)$ be a function of $t$ alone and let $\left(g_{t}, k_{t}\right) \in \mathfrak{M} \times S_{2}(M)$ be a solution of the Einstein system with $N_{t}=N(t), X=0$. Let

$\tau(t)=\int_{0}^{t} N(\lambda) d \lambda$ and let $\tau^{-1}(t)$ be its inverse. Then

$$
\bar{g}_{t}=g \circ \tau^{-1}(t) \text { and } \quad \vec{k}_{t}=k \circ \tau^{-1}(t)
$$

is the solution to $(\mathrm{E})$ with $N_{t}=1$ and

$$
\left(\bar{g}_{0}, \bar{k}_{0}\right)=\left(g_{0}, k_{0}\right)
$$

Remark: $\bar{g}_{\tau(t)}=g_{t}$ and $\bar{k}_{\tau(t)}=k_{t^{*}}$

Proof: That $\left(\bar{g}_{0}, \bar{k}_{0}\right)=\left(g_{0}, k_{0}\right)$ follows from $\tau^{-1}(0)=0$. Also,

$$
\begin{aligned}
& \frac{d \bar{g}(t)}{d t}=\frac{d g}{d t}\left(\tau^{-1}(t)\right) \frac{d \tau^{-1}}{d t}(t)=(N k)\left(\tau^{-1}(t)\right)\left(\frac{d \tau}{d t}\left(\tau^{-1}(t)\right)\right)^{-1} \\
& \text { and }
\end{aligned}
$$

$$
\begin{aligned}
\frac{d \bar{k}(t)}{d t}=\frac{d k}{d t}\left(\tau^{-1}(t)\right) \frac{d \tau^{-1}}{d t}(t)=S_{g_{\left(\tau^{-1}(t)\right)}}\left[k\left(\tau^{-1}(t)\right)\right] \\
-2 \operatorname{Ric}\left[g\left(\tau^{-1}(t)\right)\right]=S_{\bar{g}_{t}}(\bar{k}(t))-2 \operatorname{Ric}(\bar{g}(t))
\end{aligned}
$$

so that $\left(\bar{g}_{t}, \bar{k}_{t}\right)$ is a solution to $(\mathrm{E})$ with $\bar{N}_{t}=1$.

Now we briefly consider the converse program; namely, given the solution $\left(g_{t}, k_{t}\right)$ to $(E)$ with lapse $N_{t}=1$, and given an arbitrary lapse $N_{t}$, find the solution $\left(\bar{g}_{t}, \bar{k}_{t}\right)$ to $(\mathrm{E})$ with lapse $N_{t}$ and such that $\left(g_{0}, k_{0}\right)$ $=\left(\bar{g}_{0}, \bar{k}_{0}\right)$.

We claim that by a simple trick this program can be carried out by solving for the Gaussian coordinates of a suitably altered space-time.

Theorem 10.3: Let $\left(g_{t}, k_{t}\right) \in \mathrm{M} \times \mathrm{S}_{2}(M),|t|<\epsilon$ be a solution of the Einstein system (E) with lapse $N_{t}=1$, $X_{t}=0$. Let $N_{t}$ be a given lapse function. Construct a Lorentz metric on $I \times M$ by setting

$$
l_{\alpha \beta}=\frac{-d t^{2}}{N^{2}}+\frac{g_{i j}}{N^{2}} d x^{i} d x^{j}
$$

and let $\bar{x}^{\mu}\left(x^{\alpha}\right), \bar{t}\left(0, x^{i}\right)=0, \bar{x}^{i}\left(0, x^{j}\right)=x^{i}$ be the transformation of $l_{\alpha \beta}$ to Gaussian coordinates. In these new coordinates, the metric $g_{\alpha \beta} d x^{\alpha} d x^{\beta}=-d t^{2}$ $+g_{i j} d x^{i} d x^{j}$ is transformed to $\bar{g}_{\alpha \beta} d \bar{x}^{\alpha} d \bar{x}^{\beta}=-N^{2} d \bar{t}^{2}+$ $\bar{g}_{i j} d \bar{x}^{i} d \bar{x}^{j}$, so that $\left(\bar{g}_{t},\left(1 / N_{t}\right)\left(\partial \bar{g}_{t} / \partial t\right)\right),|t|<\epsilon^{\prime} \leq \epsilon$, $\bar{g}_{t}=\bar{g}_{i j}$ solves (E) with lapse $N_{t}$ and $\left(\bar{g}_{0}, \bar{k}_{0}\right)=\left(g_{0}, k_{0}\right)$.

Proof: The conditions that the new coordinates $\bar{x}^{\mu}\left(x^{\alpha}\right)$ transform $l_{\alpha \beta}$ to Gaussian coordinates are

$$
\left\{\begin{array}{c}
-1=-N^{2}\left(\frac{\partial \bar{t}}{\partial t}\right)^{2}+N^{2} g^{i j} \frac{\partial \bar{t}}{\partial x^{i}} \frac{\partial \bar{t}}{\partial x^{j}} \\
0=-N^{2} \frac{\partial \bar{t}}{\partial t} \frac{\partial \bar{x}^{i}}{\partial t}+N^{2} g^{k l} \frac{\partial \bar{t}}{\partial x^{k}} \frac{\partial \bar{x}^{i}}{\partial x^{l}} .
\end{array}\right.
$$

For any $g_{i j}$, we can solve these partial differential equations for $\bar{x}^{\mu}\left(x^{\alpha}\right)$ with initial conditions $\bar{x}^{\mu}\left(0, x^{i}\right)$ $=\left(0, x^{i}\right)$ by Lemma 10.1 and Theorem 10.1 .

The conditions that a coordinate transformation $\bar{y}^{\mu}\left(x^{\alpha}\right)$ transform $g_{\alpha \beta} d x^{\alpha} d x^{\beta}=-d t^{2}+g_{i j} d x^{i} d x^{j}$ to $\bar{g}_{\alpha \beta} d \bar{x} \alpha d \bar{x}^{\beta}=-N^{2} d \bar{t}^{2}+\bar{g}_{i j} d \bar{x}^{i} d \bar{x}^{j}$ are 


$$
\left\{\begin{aligned}
-\frac{1}{N^{2}} & =-1\left(\frac{\partial \bar{y}^{0}}{\partial t}\right)^{2}+g^{i j} \frac{\partial \bar{y}^{0}}{\partial x^{i}} \frac{\partial \bar{y}^{0}}{\partial x^{j}}, \\
0 & =-1 \frac{\partial \bar{y}^{0}}{\partial t} \frac{\partial \bar{y}^{i}}{\partial t}+g^{l} \frac{\partial \bar{y}^{0}}{\partial x^{k}} \frac{\partial \bar{y}^{i}}{\partial x^{i}} .
\end{aligned}\right.
$$

But Eqs. (10.5) are equivalent to (10.4).

We remark that by our above work, we know that when transforming $l_{\alpha \beta}$ to Gaussian coordinates, the functions $\tau_{t}(m)=\bar{t}\left(t, x^{i}\right)$ and $\phi_{t}(m)=\bar{x}^{i}\left(t, x^{j}\right)$ satisfy

$$
\left\{\begin{array}{l}
\frac{d \phi_{t}}{d t}=\sqrt{\frac{1}{N^{2}}+\left\|\operatorname{grad} \tau_{t}\right\|^{2}}, \\
\frac{d \phi_{t}}{d t} \cdot \phi_{t}^{-1}=-\phi_{*}\left(-\frac{\operatorname{grad} \tau}{d \tau / d t}\right),
\end{array}\right.
$$

so that if $\psi_{t}$ is the flow of $-\operatorname{grad} \tau /(d \tau / d t), \phi_{t}=\psi_{t}^{-1}$. Equations (10.6) are just (10.4) rewritten.

Also, as in Theorem 10.2 the equations for $\bar{g}_{t}$ are given by

$\bar{g}^{-1}\left(\tau_{t}(m), \phi_{t}(m)\right)=T \phi_{t} \otimes T \phi_{t}\left(g_{t}^{-1}-\frac{\operatorname{grad} \tau}{d \tau / d t} \otimes \frac{\operatorname{grad} \tau}{d \tau / d t}\right)$,

which follows from the coordinate expression

$\bar{g}^{i j}\left(\bar{x}^{\lambda}\left(x^{\alpha}\right)\right)=\frac{\partial \bar{x}^{i}}{\partial x^{k}}\left(x^{\alpha}\right) \frac{\partial \bar{x}^{j}}{\partial x^{l}}\left(x^{\alpha}\right)^{\prime} g^{k l}\left(x^{\alpha}\right)-\frac{\partial \bar{x}^{i}}{\partial t}\left(x^{\alpha}\right) \frac{\partial \bar{x}^{j}}{\partial \bar{t}}\left(x^{\alpha}\right)$

and (10,4).

\section{THE RELATIONSHIP OF THE MANIFOLD $a$ TO SUPERSPACE AND SOME REMARKS ON SUPERPHASE SPACE}

Let

$$
\Phi: \mathcal{D} \times \mathfrak{M} \rightarrow \mathfrak{K}, \quad(\eta, g) \mapsto\left(\eta^{-1}\right)^{*} g
$$

be the left action of $D$ on $\mathfrak{T}$. Then superspace $S(M)$, or the space of all geometries of $M$, is defined as the orbit space $\mathfrak{T} / D$ of this action. This is explained briefly as follows: For fixed $g \in \mathfrak{M}$, let

$$
\theta_{g}=\left\{\left(\eta^{-1}\right)^{*} g \mid \eta \in \mathbb{D}\right\} \subset \mathfrak{M}
$$

be the orbit of $D$ through $g$. Then $\theta_{g}$ is the set of all metrics isometric to $g$. Since $S(M)$ is the set of all orbits in $M$,

$$
S(M)=\mathfrak{M} / \mathfrak{D}=\left\{\theta_{g} \mid g \in \mathfrak{N}\right\},
$$

$S(M)$ is the space of all isometry classes of Riemannian metrics or geometries on $M$.

The importance of $S(M)$ is that it is the natural configuration space for a dynamical theory of general relativity. The reason for this is that isometric Riemannian metrics are physically indistinguishable; thus a physical state determines only an isometry class of Riemannian metrics. In the language of the classical physicist, the metric representing the physical state is determined only up to a coordinate transformation.

Unfortunately $S(M)$ is not a differentiable manifold. This is because the isometry group $I_{g}=\left\{\eta \in \mathcal{D} \mid\left(\eta^{-1}\right)^{*}\right.$ $g=g\}$ of a metric $g \in \mathfrak{M}$ is different for different $g$. As the isometry group $I_{f}$ is the isotropy group of the action $\Phi$ at $g$, the resulfing orbit space is not a manifold; in other words, the symmetric geometries do not have neighborhoods homeomorphic to neighborhoods of geometries which have no symmetries whatsoever. $S(M)$ can, however, be stratified into differentiable manifolds, each strata $\delta_{G)}(M)$ being all those geometries whose isometry groups $I_{g}$ determine equivalent group actions on $M$. This, however, is a rather long story; for details see Ref. 5 .

Since $S(M)$ is not a manifold, it is awkward to use $S(M)$ as the configuration space for a dynamical system. This difficulty becomes apparent as soon as we try to construct the tangent bundle $T(\mathscr{T} / \mathcal{D})$, the velocity phase space. It is probably possible to give meaning to $T(\mathfrak{T} / D)$ by taking limits of tangent spaces and using the notion of tangent cones. However, the singularities of $S(M)$ would then be severely compounded.

A way to short circuit this approach is to define as the superphase space not $T(\mathcal{T} / / D)$, but rather

$$
(T \mathscr{M}) / \mathscr{D}=\frac{\mathscr{T} \times S_{2}(M)}{\mathbb{D}} \neq \frac{\mathscr{T}}{\mathscr{D}} \times \frac{S_{2}(M)}{\mathscr{D}}
$$

the orbit space of the action

$$
\Phi^{\prime}: \mathbb{D} \times T \mathscr{T} \rightarrow T \mathscr{K}, \quad(\eta, g, k) \mapsto\left(\left(\eta^{-1}\right)^{*} g,\left(\eta^{-1}\right)^{*} k\right) .
$$

Note that $T M / D$ is not equivalent to $T(T / D)$. $T \mathfrak{T} / D$ is perhaps a more likely candidate for superphase space as the tensor fields $(g, k) \in T \mathfrak{N}$ are subjected only to the same active coordinate transformation; that is, the pair $(g, k)$ must transform together. From Corollary 4.1 we see that this is appropriate from the dynamical point of view:

Unfortunately, $T \mathscr{M} / \mathcal{D}=\left(\mathfrak{T} \times S_{2}(M)\right) / \mathcal{D}$ suffers from all of the pathologies that $S(M)$ does, as well as the added difficulties related to the structure of $S_{2}(M) / D$. Note for example that $D$ leaves invariant the 0 -tensor field in $S_{2}(M)$, so that the isotropy subgroup of 0 need not even be finite-dimensional (as it is for $\Phi, \Phi^{\prime}$ ). Thus the construction of equations of motion on $T$ T/D directly does not seem feasible at this time. Nevertheless, the dynamics on TIT, followed by a projection onto $T M T / D$ does recapture all the essential elements that a dynamical system on $T \mathfrak{T} / D$ would have to possess. In fact, $T \pi / D$ inherits a continuous flow from the flow on $T \mathrm{~T}$. Thus we have a $\mathrm{C}^{0}$ or topological dynamical system.

To incorporate the shift vector field into the dynamics we have in the course of this paper chosen $T(D \times M)$ as the velocity phase space. The degeneracy in our Lagrangian allows one to specify arbitrarily a curve $\eta_{t}, \eta_{0}=i d_{M}$, in the factor $D$ or equivalently a shift vector field $X_{t}$. The solution $\left(g_{t}, k_{t}\right)$ of $(E)$ with shift vector field $X_{t}$ is then related to a reference solution $\left(\bar{g}_{t}, \bar{k}_{t}\right)$ of $(\mathrm{E})$ with shift $\bar{X}_{t}=0$ by $\left(g_{t}, k_{t}\right)=\left(\left(\eta_{t}^{-1}\right)^{*} \bar{g}_{t}\right.$, $\left.\left(\eta_{t}^{-1}\right)^{*} \bar{k}_{t}\right)$. Thus $\left(g_{t}, k_{t}\right)$ can be thought of as $\left(\bar{g}_{t}, \bar{k}_{t}\right)$ sliding along the orbits $\theta_{\left(g_{t} k_{t}\right)}=\left\{\left(\left(\eta^{-1}\right)^{*} g,\left(\eta^{-1}\right)^{*} k\right) \mid \eta\right.$ $\in D\} \subset \mathscr{N} \times S_{2}(M)$. The factor $D$ then keeps track of the amount of sliding relative to the reference curve $\left(\bar{g}_{t}, \bar{k}_{t}\right)$.

Note that the curve $\left(g_{t}, k_{t}\right)$ and $\left(\bar{g}_{t}, \bar{k}_{t}\right)$ are projected onto the same curve in $T M / D$ as we have divided out by $D$, so that solutions to $(E)$ which differ only by a shift vector field map to the same curve in $T T / D$. Thus if we could construct a dynamical system on $T \mathfrak{M} / \mathcal{D}$ directly, it would be independent of the shift. 
We also remark that if we define $\left(\eta_{1}, g_{1}\right) \in \mathbb{D} \times \mathfrak{M}$ to be equivalent $(=\sim)$ to $\left(\eta_{2}, g_{2}\right) \in \mathscr{D} \times \mathfrak{M C}$ if $\left(\eta_{1}^{-1}\right)^{*} g_{1}$ $=\left(\eta_{2}^{-1}\right)^{*} g_{2}$, then $D \times \Re / \sim \cong \Re / D$. Similarly, if on $\mathcal{T}(D \times \mathscr{T})$ we define $\left(X_{\eta_{1}}, g_{1}, k_{1}\right) \sim\left(Y_{\eta_{1}}, g_{2}, k_{2}\right)$ if $\left(\left(\eta_{1}^{-1}\right)^{*} g_{1},\left(\eta_{1}^{-1}\right)^{*} k_{1}\right)=\left(\left(\eta_{2}^{-1}\right)^{*} g_{2},\left(\eta_{2}^{-1}\right)^{*} k_{2}\right)$, then $T(D) \times$ $\mathscr{T C}) / \sim=T \mathrm{~T} / \mathrm{D}$. Thus we recapture the space of physically indistinguishable states $T \mathbb{T} / D$ by defining a suitable equivalence relation on $T Q \approx T(D) \times T)$. Of course this equivalence relation is natural from the point of view of the dynamical development of the states.

We now wish to incorporate the lapse function into the picture. For nonrelativistic classical field theories, there is a canonical parameter of evolution, namely the time $t$. For covariant relativistic field theories in general, and for general relativity in particular, the proper time $\tau$ plays the role of a canonical parameter of evolution. In order to maintain covariance, however, one must allow for an arbitrary reparameterization of this evolution parameter. This reparameterization may also depend on the space points of the field. It is because of this possible space dependence of the change of parameter that Wheeler refers to time as a many fingered entity; this is associated with the DiracTomonaga-Schwinger many time formalism for quantum field theory.

Another well-known implication of covariance is that a covariant field theory when expressed in a dynamical formulation must be degenerate. This situation comes about because the resulting dynamics must be able to be summed up as a tensor field on a four dimensional manifold $V$. Each slicing of $V$, therefore, gives rise to a different dynamical system all of which are equivalent in the sense that they lead to the same tensor field on $V$. As the dynamical formalism must take into account this arbitrary slicing, it must be degenerate. In this paper the introduction of $\tau$ accounts for this arbitrary slicing of a space-time; $D$ takes into account the possible coordinates in each slice. Let $V=I \times M$, and let $\{t\} \times M$ be the $t=$ constant hypersurfaces. Let $\tau_{t}$ be a curve in $\tau$ and let $\tau: I \times M \rightarrow \mathbf{R},(t, m) \mapsto \tau_{t}(m)$. Then $\tau^{-1}(t)$ are the $\tau=$ constant hypersurfaces. Thus each curve $\tau_{t} \in \mathcal{T}$ maps $t=$ constant to $\tau=$ constant hypersurfaces and thus can be considered as an arbitrary slicing of $V$ with respect to some reference slicing which represents $V$ as $I \times M$.

Let $\left(\bar{g}_{t}, \bar{k}_{t}\right)$ be a solution of $(\mathrm{E})$ with $\bar{X}=0, \bar{N}=1$. We consider the curve $\tau_{t} \in \mathcal{T}$ defined by $\bar{\tau}_{t}(m)=1$ as a reference curve. We construct the space-time $g_{\alpha \beta} d x^{\alpha} d x^{\beta}=-d t^{2}+g_{i j} d x^{i} d x^{j}$ on $I \times M$. Now suppose that we are given an arbitrary curve $\tau_{t} \in T, \tau_{0}=0$. Alternately, by Lemma 10.1 we may suppose we are given some lapse function $N_{t}$ and then find $\tau_{t}$ associated with it and $\bar{g}_{t}$. The evolution for this new lapse or new $\tau_{t}$ is determined by finding the metric on the $\tau=$ constant hypersurfaces. Of course, these evolutions are equivalent in that they determine isometric Lorentz metrics or, in other words, are summed up by the same space-time. Nevertheless, given a curve $\left(\bar{g}_{t}, \bar{k}_{t}\right) \in T \mathfrak{T}$, each curve $\tau_{t}$ determines a dynamical curve $\left(g_{t}, k_{t}\right) \in T M$. Thus we map curves in $\mathcal{T}$ to curves in $T T / D=T(D \times T C) / \sim$. The image in $S(M)$ $\times S_{2}(M) / \mathcal{D}$ of all curves in $\mathcal{T}$, projected onto $S(M)$, is just the sheaf in superspace which summarizes the space-time. This idea is described by DeWitt ${ }^{23}$ without the use of the space $\mathcal{T}$.

\section{CONCLUSIONS AND FURTHER WORK}

In this paper we have attempted to clarify the Hamiltonian structure of the Einstein equations and to achieve a clear understanding of the geometrical roles played by the lapse and shift functions. We feel that we have gained a more natural form for the phase space of general relativity by introducing the groups $D$ and $\mathcal{T}$. For example, by enlarging the configuration space from $\mathfrak{T}$ to $T \times D \times \mathfrak{D}$, the lapse and shift functions may be incorporated into the dynamics as dynamical velocities. Moreover, we showed explicitly how one can obtain solutions for any lapse or shift from the trivial ones $N=1, X=0$ by integrating a system of ordinary differential equations. In connection with the lapse, we introduced a new object, the intrinsic shift, which takes into account the spatial shifting of the $\{t\} \times M$ hypersurfaces when mapped into $\tau=$ constant hypersurfaces.

We feel that the introduction of the groups $D$ and $\mathcal{T}$ helps to properly understand the basic conservation laws for $\delta \pi$ and $\mathcal{H}$ as a consequence of dynamical symmetries. On the other hand, we are forced to accept a degenerate Lagrangian system. This degeneracy is present and is perfectly natural when one considers any covariant field theory from a dynamical point of view.

Some work which remains to be done is to explore whether or not the procedure presented here helps to clarify any of the difficult quantization problems. However, preliminary indications are that quantization problems run much deeper. For example, in the usual quantum theory of fields one deals with equations of the form $\square \phi+F(\phi)=0$, for definiteness say $\square \phi+\lambda \phi^{3}=m^{2} \phi$. As a classical partial differential equation, this equation is semilinear, as the highest order derivatives occur linearly, the nonlinearity occurring only in the $\phi^{3}$ term. As is well known, a rigorous and complete quantization of such equations is very difficult and, in fact, has not yet been achieved for four-dimensional space-times. In relativity, the basic structure of the evolution equations is quite different. Let us, for example, neglect the fact that the equations for a space-time are a system of partial differential equations. Then, roughly speaking, the "scalar analog" of the evolution equations is the quasilinear equation $\phi \square \phi+\|\operatorname{grad} \phi\|^{2}=0$, $\operatorname{grad} \phi=g^{\alpha \beta}\left(\partial \phi / \partial x^{\alpha}\right)$. Now $\phi$ itself is involved in the coefficients of the operator $\phi \square$. Also, the equation involves nonlinear derivative coupling terms. Very little is known about the quantization of such an equation. Moreover, the equations for relativity are much more involved, as they involve a system of quasilinear equations, the components of which are very badly mixed in the highest order (unless one chooses the harmonic coordinate condition) and first-order derivative terms. Thus, a complete quantum theory of general relativity seems quite far away. ${ }^{3,24}$

In a for thcoming paper 18,14 we shall be focussing our attention on problems of existence and uniqueness of 
solutions using the theory of quasilinear first-order symmetric hyperbolic systems, rather than the usual methods using the considerably more complicated theory of second-order strictly hyperbolic systems. Our existence proof will follow simply and directly from such a first-order treatment. Moreover, we will be able to give a more intrinsic treatment by making use of a global version of the symmetric hyperbolic theory.

\section{ACKNOWLEDGMENTS}

We thank P. Chernoff, D. Ebin, H. Kunzle, K. Kuchar̆, R. Sachs, and A. Taub for a variety of helpful sugges tions.
* Partially supported by AEC Contract AT(04-3)-34.

+ Partially supported by Navy Contract N00014-69-A-0200-1002 and NSF Contract GP-8257. Part of this author's work was done while at the University of Toronto, Toronto, Canada, and the Institute for Advanced Study, Princeton, New Jersey.

1 J.A. Wheeler, "Geometrodynamics and the Issue of the Final State," in Relativily, Groups and Topology, edited by B. DeWit and C. DeWitt (Gordon and Breach, New York, 1964).

2 R. Arnowitt, S. Deser, and C. W. Misner, "The Dynamics of General Relativity," in Gravilation: An Iniroduction to Current Research, edited by L. Witten (Wiley, New York, 1962).

3 B. DeWitt, Phys. Rev. 160, 1113 (1967).

C. W. Misner, Rev. Mod. Phys. 29, 497 (1957).

5 A. Fischer, "The Theory of Superspace," in Relativity. edited by M. Carmeli, S. Fickler, and L. Witten (Plenum, New York, 1967).

6 R. Arnowitt, S. Deser, and C. W. Misner, Phys. Rev. 116, 1322 (1959).

7 V. Arnold, Ann. Inst. Fourrer (Grenoble) 16, 319 (1966); D. Ebin and J. Marsden, Ann. Math. 92, 102 (1970)

8 R. Abraham, Founciations of Mechanics (Benjamin, New York, 1967).

9 C. Lanczos, The Varialional Principles of Mechanics (U. of Toronto Press, Toronto, 1966), 3rd ed.

10 D. Ebin, "The Manifold of Riemannian Metrics," in Proceedings of the Symposium on Pure Mathematics (Amer. Math. Soc., Providence, R. I., 1970), Vol. 15.

11 J. Marsden, Arch. Ratl. Mech. Anal. 28, 362 (1968).

12 S. Lang, Introduction to Differentiable Manifolds (Wiley, New
York, 1962).

13 Y. Choquet-Bruhat, Compt. Rend. 272, 386 (1971); Ann. Inst. H. Poincaré 8, 327 (1968).

14 A. Fischer and J. Marsden, "The Einstein Evolution Equations as a Quasi-Linear First Order Symmetric Hyperbolic Sy stem. I" (to be published)

15 D. Eardley, E. Liang, and R. Sachs, J. Math. Phys. 13, 99 (1972).

16 S. Deser, Ann. Inst. H. Poincaré 7, 146 (1967); M. Berger and D. Ebin, J. Diff. Geom. 3, 379 (1969).

17 A. Lichnerowicz, Relativislic Hydrodynamics and Magnelohydrodynamics (Benjamin, New York, 1967).

18 A. Fischer and J. Marsden, "General Relativity, Partial Differential Equations, and Dynamical Systems," in Proceedings of the Symposium on Pure Mathematics (Amer. Math. Soc Providence, R. I., 1972), Vol. 23.

19 Y. Choquet-Bruhat and R. Geroch, Commun, Math. Phys. 14, 328 (1969).

20 D. Brill and S. Deser, Ann. Phys. (N.Y.) 50, 548 (1968); J. Marsden and A. Fischer, "The Existence of Complete Spacetimes" (in preparation).

$21 \mathrm{~K}$. Yano, Inlegral Formulas in Riemannian Geomelric (Decker, New York, 1971).

22 R. Abraham, J. Math. Mech. 11, 553 (1962).

23 B. DeWitt, "Space-Time as a Sheaf of Geodesics in Superspace," in Relativily, edited by M. Carmeli, S. Fickler, and L. Witten (Plenum, New York, 1970).

24 L. D. Faddeev, Act. Cong. Intern. Math. 3,35 (1970).

\section{States, Minimizing the Uncertainty Product of the Oscillator Phase Operators}

\section{Evangelos K. Ifantis}

Nuclear Research Center "Democritos", Aghia Paraskevi, Attikis, Athens, Greece

(Received 8 October 1971; Revised Manuscript Received 29 November 1971)

The normalizable states that minimize the uncertainty product of the oscillator phase operators are determined and some of their physical properties are discussed. A physical classification of these states has been made and the class of "analogous" states to the well-known coherent states is physically defined.

\section{INTRODUCTION}

Quantum mechanically it is convenient to define the phase $\phi$ of the harmonic oscillator indirectly by defining the "cosine" and "sine" operators $C$ and $S$, which correspond to $\cos \phi$ and $\sin \phi$ in the classical limit. The operators $C$ and $S$ found do not commute, i.e., the $\cos \phi$ and $\sin \phi$ cannot be measured simultaneously. It is therefore interesting to find the normalizable states, which minimize the uncertainty product $(\Delta C)^{2} \cdot(\Delta S)^{2}$ of $C$ and $S$.

It was proved in Ref. 1 and was noted in further re$\operatorname{search}^{2,3}$ on the quantum mechanical oscillator phase problem that there exist no normalizable states that minimize the uncertainty product $(\Delta C)^{2 \cdot(\Delta S)^{2}}$. This result is correct in the sense that for these states the inequality $(\Delta C)^{2}(\Delta S)^{2} \geqslant \frac{1}{4}\langle(1-p) / 2\rangle^{2}$ becomes an equality and, moreover, $\langle 1-p\rangle^{2}$ becomes a greatest lower bound.

We have shown in a previous work, ${ }^{4}$ using methods of the spectral theory of bounded operators, that normalizable states minimizing the uncertainty product
$(\Delta C)^{2 \cdot(\Delta S)^{2}}$ do exist in the sense that the above inequality becomes an equality.

In the present work we determine these states and find some of their physical properties. Moreover, we classify the normalizable minimal uncertainty states and characterize, both mathematically and physically, the states that have properties analogous to those of the well known coherent states.

In Sec. 2 we present some general properties of the normalizable minimal uncertainty states and the "minimal uncertainty sequences" of states for arbitrary noncompatible observables $A$ and $B$. We note that the knowledge of the point spectrum and the continuous spectrum of the non-self-adjoint operator $A+i_{\gamma} B$ is sufficient for the determination of the expectation values of $A$ and $B$ in the corresponding states. The determination is exact in the case of the point spectrum and approximate in the case of the continuous spectrum. In addition we characterize mathematically the states that have properties analogous to those of the coherent states. 